Archives de sciences sociales des religions

138 | avril - juin 2007

Varia

\title{
La théologie de la libération en Amérique latine
}

Approche sociologique

Malik Tahar Chaouch

\section{CpenEdition}

Journals

Édition électronique

URL : http://journals.openedition.org/assr/4822

DOI : $10.4000 /$ assr.4822

ISSN : $1777-5825$

Éditeur

Éditions de l'EHESS

Édition imprimée

Date de publication : 1 juin 2007

Pagination : 9-28

ISBN : 978-2-7132-2143-9

ISSN : 0335-5985

Référence électronique

Malik Tahar Chaouch, «La théologie de la libération en Amérique latine », Archives de sciences sociales des religions [En ligne], 138 | avril - juin 2007, mis en ligne le 05 septembre 2010, consulté le 10 décembre 2020. URL : http://journals.openedition.org/assr/4822 ; DOI : https://doi.org/10.4000/assr. 4822 


\section{Malik Tahar Chaouch}

\section{La théologie de la libération en Amérique latine Approche sociologique}

Depuis les années 1970, le thème de la théologie de la libération en Amérique latine a suscité une volumineuse littérature dans les sciences sociales. Le radicalisme de son option pour le changement social passionne. Elle témoigne notamment de l'engagement de chrétiens, en majorité catholiques, en faveur du socialisme. Dans le contexte de la post-guerre froide, le silence se fait progressivement autour d'elle. Pourtant, l'impact actuel de dynamiques et d'imaginaires sociaux, situés dans sa trajectoire, est incontestable. Le mouvement zapatiste au Mexique et les forums sociaux mondiaux de Porto Alegre n'en sont que les manifestations les plus visibles.

La plupart des recherches antérieures associaient la théologie de la libération à la notion indifférenciée d'un vaste mouvement social. Nous avons opté pour la reconstruction critique d'un champ social. Sans nier ses liens avec des dynamiques sociales et politiques, cette approche conduit à rendre compte des stratégies et intérêts différenciés des acteurs investis dans ces dynamiques. La littérature sur le thème en soumettait l'interprétation à la reprise des idées de la théologie de la libération qui présupposent un sujet populaire de transformation sociale, inspiré par sa foi religieuse. Notre démarche permet de recentrer l'analyse sur les acteurs réels, au détriment de généralités idéologiques largement démenties par la réalité.

Le rapport ambigu des agents catholiques de la théologie de la libération au pouvoir ecclésial constitué et à son idéologie est mis ici en évidence ; sont dessinés les traits d'un type intellectuel, dont l'identification au sujet populaire, censé fonder sa réflexion, est très discutable. Enfin, par-delà la référence absolutisée aux contextes sociopolitiques de la région, est montré le rôle central joué par les réseaux transnationaux, catholiques et œcuméniques, qui circulaient entre l'Europe, l'Amérique du Nord et l'Amérique latine ${ }^{1}$.

1. Notre enquête de terrain s'est concentrée dans les années 2000-2001 sur les lieux de passage de ces réseaux au Costa Rica, au Mexique et en Colombie. Elle a bénéficié, au Costa Rica, de la participation directe à un séminaire au sein du DEI (Departamento Ecuménico de Investigación), acteur privilégié de ces réseaux. L'analyse s'appuie sur un corpus de quarante entretiens, complété par la consultation de sources écrites dans quinze organisations religieuses, civiles et œcuméniques. 


\section{Premiers éléments}

On peut percevoir la faiblesse de l'analyse sociologique de la littérature militante sur la théologie de la libération (Oliveiros, 1977 ; Silva Gotay, 1981 ; Berrymann, 1987 ; Del Valle, 1996 ; Concha, 1997) dans sa réduction de la réalité sociale du phénomène à un combat bipolaire entre catholicisme " officiel » et «Église des pauvres". Le premier, accusé de complicité avec la situation latino-américaine d'injustice et de misère sociale, et la seconde, censée se fonder sur l'alliance entre l'action conscientisatrice de ses agents ecclésiaux et ses bases populaires conscientisées. Son combat déclaré pour la justice sociale implique une série d'oppositions symétriques, non moins simplistes : entre son progressisme et le conservatisme catholique ; entre l'émergence d'un projet ecclésial construit «depuis en bas » et le repli de l'institution sur son principe vertical d'autorité ; entre une théologie proprement latino-américaine et la théologie européenne ; entre une religion libératrice et l'aliénation religieuse. Cette littérature reprend à son compte les notions d' "Église populaire " et d' "Église progressiste » (Levine, Mainwaring, eds., 1986 ; Mainwaring, Wilde, eds., 1988 ; Levine, 1992 ; Hewitt, Burdick, eds., 2000). L’hostilité à la théologie de la libération ne dépasse pas le niveau de la polémique et demeure cantonnée dans le débat idéologique. Elle se contente de contester le caractère «chrétien » de la théologie "marxiste " de la libération et de s’inquiéter de la "réduction politique " de la signification des Évangiles (Lepeley, 1985 ; Jiménez, 1988 ; Bigo, 1990 ; Lynch, 1990 ; Cierva y de Hoces, 1996).

En revanche, d'autres textes avancent des éléments de déconstruction du discours de justification de la théologie de la libération. Ils interrogent la réalité «populaire » et l'autonomie des communautés ecclésiales de base, soumises au contrôle de l'Église catholique (Lehmann, 1996). Ils questionnent le rapport entre ces communautés et la théologie de la libération, comme discours intellectuel d'élites catholiques «transnationalisées "(Corten, 1995 : 15-44). L'imaginaire "basiste » de la gauche brésilienne, dont participe ce discours, y est clairement distingué d'un imaginaire populaire (pp. 99-134). La distance entre l'idéal radical de transformation sociale et la réalité, souvent plus traditionnelle, des pratiques religieuses au sein des communautés ecclésiales de base est soulevée par un analyste de l' «Église populaire» (Levine, 1990)². En ce sens, surgit un intérêt récent pour les dynamiques transcontinentales de réseaux militants (Colonomos, 2000, pp. 59-69 ; Corten, Matas, Sauvage, 2001). Enfin, des approches sociohistoriques suivent la piste du catholicisme intransigeant en Amérique latine, défini par Émile Poulat comme un catholicisme d'opposition aux sociétés modernes et sécularisées (Poulat, 1977). Elles ouvrent timidement la voie vers l'hypothèse du poids

2. Cet article fut publié dans Archives des Sciences Sociales des Religions, 71, dont le dossier était consacré à la théologie de la libération en septembre 1990. L’article de Michael Löwy, dans ce même numéro, a été repris dans son ouvrage de 1998, auquel nous faisons référence, 
de cette généalogie dans les ambivalences du "progressisme » du catholicisme latino-américain (Mallimaci, 1988 ; Blancarte, 1992 ; Meyer, 2000). Toutes ces pistes méritent d'être réunies et approfondies dans un cadre intégré et systématique de réinterprétation du phénomène.

\section{La théologie de la libération comme mouvement social}

À ce jour, l'interprétation scientifique systématique la plus consolidée consiste à définir la théologie de la libération comme l'expression d'un mouvement social (Muro González, 1982, 1991, 1994 ; Smith, 1991 ; Löwy, 1998), en renonçant à des appellations plus classiques pour le désigner, comme celle d'« Église populaire ». Pour Michael Löwy, citant Leonardo Boff, l'idée provient directement des théologiens de la libération : attachés à l'idée de "praxis ", ils la considèrent comme «(...) le reflet d'une pratique qui lui est antérieure et une réflexion sur celle-ci» (Löwy, 1998, 53). Plus précisément, Löwy la définit comme « (...) l'expression d'un mouvement social qui s'est dégagé au début des années 1960 - bien avant que ne paraissent les nouveaux ouvrages de théologie » (p. 53) et qu'il qualifie de "christianisme de la libération».

Ce passage conceptuel à la notion de "mouvement social » implique une discontinuité partielle avec les interprétations apologétiques antérieures et rompt avec le discours simpliste d'opposition symétrique construite autour de l'idée d' "Église populaire ». Ainsi, Löwy s'oppose à la thèse d'un mouvement « de la base vers le sommet " de l'Église catholique, autant qu'à la thèse inverse. Il préfère envisager un mouvement " de la périphérie vers le centre » de l'institution (p. 65) et insiste sur le caractère périphérique des acteurs catholiques qui animaient le mouvement envisagé par lui : prêtres et mouvements laïcs engagés dans les quartiers populaires, experts laïcs des commissions ecclésiales, ordres religieux et missionnaires étrangers.

Sur le cas mexicain, Víctor Gabriel Muro González soutient une thèse semblable $^{3}$ rejetant le simplisme de la division entre deux modèles d'Église, l'un «populaire » et l'autre attaché à l'idée de chrétienté. Il n'accepte pas la réduction de la théologie de la libération à une simple stratégie d'influence de l'Église catholique contre l'hégémonie de l'État mexicain dans la vie politique nationale et rappelle que les évêques mexicains, comme le Vatican, étaient majoritairement hostiles à cette politisation. Pour lui, la théologie de la libération est l'expression des liens construits entre des secteurs de l'Église catholique et de la société civile nationale et qui ont réorienté la pastorale dans un sens politique, l'obligeant à centrer l'analyse sur la corrélation entre la théologie de la libération et le changement social. Cette corrélation représente un double défi pour l'hégémonie étatique et les stratégies dominantes de l'Église catholique. Toutefois, Muro ne nie

3. Nous résumons ici la thèse qu'il présenta de façon synthétique dans son article de 1992. 
pas la légitimité qu'en tirent, dans un contexte de mutation sociale, les secteurs ecclésiaux ainsi engagés.

Pour sa part, Christian Smith tente de systématiser au maximum la vision de la théologie de la libération comme l'expression d'un mouvement social, en lui appliquant le modèle d'analyse de Charles Tilly, modèle qui le conduit à préciser l'identification sociologique des acteurs individuels. Il y relève le statut clérical des principaux théologiens de la libération, leur rôle de conseillers dans des commissions ecclésiales de haut niveau et leur participation à des événements ecclésiaux d'importance (Smith, 1994, 233, trad. espagnole), ce qui relativise le caractère périphérique du mouvement. Smith met finalement l'accent sur les enjeux historiques des engagements de ces théologiens dans l'espace et la finalité d'un plus ample mouvement: les acteurs de l'Église catholique, «convertis » à l'urgence du combat social en Amérique latine, mobilisent les ressources de l'institution au bénéfice des fins transparentes de ce combat.

Sur l'essentiel, ces approches confirment les interprétations apologétiques de la théologie de la libération et rompent avec le dualisme de la littérature militante mais pas avec son finalisme. La notion indifférenciée d'un mouvement social engendre l'illusion d'une unité d'action, orientée vers l'horizon univoque du changement social. Elles reprennent ainsi ce qu'il y a de plus fondamental dans le discours de légitimation de la théologie de la libération : la conscience de l'inachèvement de la réalisation historique du sujet populaire, au nom duquel elle parle. En revanche, la vision d'une "nouvelle façon de faire la théologie » comme "réflexion critique sur la praxis historique "(Gutiérrez, 1971, p. 33) ${ }^{4}$ est placée au-dessus de tout soupçon.

\section{Une approche en terme de champ social}

L'identification de la théologie de la libération avec le mouvement envisagé rend compte de la réalité d'investissements pastoraux et militants sur le terrain de l'action collective. Elle justifie la recomposition alternative d'intérêts religieux et intellectuels exploitant la référence au mouvement. Le terme "libération ", au centre du discours, présuppose la finalité collective du changement social comme la raison d'être de la théologie de la libération. Le terme "théologie ", passé inaperçu, indique la prédominance des intérêts particuliers, religieux et intellectuels, qui s'y projettent. Il faut s'étonner davantage qu'une "théologie » puisse servir à désigner un mouvement. D’une part, on affirme la primauté du mouvement sur la réflexion théologique secondaire qui en découle : ceux qui font vraiment la théologie de la libération sont censés être les prêtres, religieux et militants engagés dans l'action. D'autre part, on contribue à l'identification légitimante d'une «théologie » avec la notion de ce mouvement: le sens de la

4. C'est l'affirmation la plus classique du texte fondateur de Gustavo Gutiérrez. 
" libération " est fixé et monopolisé par les acteurs religieux et intellectuels qui prédominent dans les conditions de cette identification.

La notion de champ social rend attentif aux modes de légitimation et intérêts sociaux de ces acteurs. Elle intègre l'analyse de leur participation aux dynamiques historiques qui justifièrent, partiellement, le recours à la notion de mouvement social. Néanmoins, elle en dépasse la conception finaliste et indifférenciée manquant à la profondeur sociologique des intérêts particuliers qui s'y projettent.

Pierre Bourdieu a défini le champ social comme un espace dont les agents luttent pour la possession de biens et de capitaux spécifiques (Bourdieu, 1984 : 113-120 ; Bourdieu, Wacquant, 1992) : par exemple du monopole de la manipulation légitime des biens du salut dans le champ religieux, structuré autour de l'opposition entre clergé et laïcs ; de l'autorité universitaire, comme forme institutionnalisée du capital culturel, dans le champ académique ; ou encore du prestige intellectuel dans le champ intellectuel, plus ample que le précédent. La distribution interne de ce capital détermine les conditions objectives de la lutte que les acteurs tendent, depuis leurs positions respectives, à conserver ou à transformer. Ceux-ci acceptent de «jouer le jeu ", intériorisant les catégories réceptives qui donnent sens à cette lutte. De ce point de vue, les acteurs dominants peuvent faire fonctionner les propriétés du champ en leur faveur et convertir les acteurs subalternes en agents de leur propre domination. Néanmoins, il existe toujours la possibilité pour ces derniers d'en contester les catégories et de modifier les rapports de force du champ. Le champ définit donc un univers singulier et hiérarchisé de pratiques et de valeurs, avec ses propres règles et logique sociales.

La définition de la théologie de la libération comme l'expression d'un mouvement social conduit à admettre, sans plus d'examen, sa valeur progressiste de rupture religieuse et la primauté de ses enjeux au sein de l'action collective, dans un environnement latino-américain de mutation sociopolitique. La reconstruction du champ déplace l'attention depuis ses bases sociales supposées vers les réseaux intéressés par ses enjeux spécifiquement religieux et intellectuels. Elle situe les limites sociales et idéologiques de son discours de rupture religieuse. Loin d'être une théologie laïque, de rupture avec le pouvoir clérical traditionnel, elle est plutôt constitutive d'un pouvoir religieux alternatif d'opposition à l'orthodoxie catholique, celui-ci, ouvert aux dynamiques laïques, étant constitué de façon décisive par une partie de la hiérarchie et du clergé. L'approche en termes de champ rend également compte des inconsistances et des usages intellectuels de son invocation de la "praxis ", en référence à un sujet populaire de libération collective. Elle s'écarte toutefois de l'idée qu'elle aurait mobilisé les ressources des champs intellectuel et religieux au bénéfice de la finalité supposée d'un vaste mouvement social. Elle tend plutôt à montrer comment la notion d'un tel mouvement permet, à l'inverse, à ses agents de s'emparer de ces ressources, sur les marges des champs impliqués. La théologie de la libération ne s'y érige plus tant comme le reflet transparent du changement social que comme une reconstruction 
idéologique de la réalité latino-américaine, au croisement de la radicalisation générationnelle des années 1960, et de la circulation des réseaux transcontinentaux du tiers-mondisme chrétien dans la région.

\section{La prédominance du clergé catholique}

Pour les besoins de sa démonstration, Löwy insiste sur le caractère périphérique des acteurs de la théologie de la libération. Ainsi, il met l'accent sur leurs solidarités militantes et dynamiques de mouvement social censées confronter, sur ses marges, le dogmatisme doctrinal et la normativité institutionnelle de l'Église catholique. L'étude du champ oblige à considérer l'étendue de ses relais et effets sociaux dans les différents contextes nationaux de l'Amérique latine. Elle tend aussi à focaliser l'analyse sur les théologiens qui prédominent. Mais, au-delà d'eux-mêmes, ces derniers cristallisent une double présence religieuse et intellectuelle, qui ne coïncide pas toujours.

Sur le plan subjectif, la théologie de la libération exalte ses bases populaires et laïques. Toutefois, son imaginaire de grandes figures de «martyrs » et de " prophètes ", invariablement issues du clergé catholique contraste avec l'anonymat des victimes ordinaires. Le premier à entrer au panthéon de ses héros tombés fut le prêtre colombien Camilo Torres, tué au combat en 1966, après s'être engagé dans la guérilla de l'ELN (Ejercito de Liberación Nacional). Suivirent l'évêque de San Salvador, Oscar Arnulfo Romero, puis les jésuites de la UCA (Universidad Centroamericana), assassinés respectivement en 1980 et 1989. À ceux-ci s'ajoutent les évêques, dont on célèbre l'exemple " prophétique », comme Sergio Méndez Arceo et Samuel Ruiz au Mexique, Leonidas Proaño en Équateur, Marco McGrath au Panama, Helder Camara, Evaristo Arns, Avelar Brandao, Aloiso Lorscheider, Candido Padin et Pedro Casaldaliga au Brésil. Au moment de la conférence épiscopale latino-américaine de Medellin en 1968, ces évêques étaient majoritaires dans la direction de la CELAM (Conferencia Episcopal Latinoamericana). Cela explique pourquoi cette dernière fut, dans un premier temps, l'un des principaux espaces de circulation et de diffusion des idées de la théologie de la libération.

L'objectivation des rapports internes du champ permet d'observer cette emprise cléricale dans un sens plus étendu. Le contenu de l'activité intellectuelle et l'intensité des engagements ne sont pas les principaux facteurs de hiérarchisation du champ. Dans un premier temps, le positionnement dans les espaces ecclésiaux est déterminant. Ainsi, la première génération de théologiens de la libération fut dominée par les conseillers de la CELAM, plus souvent clercs que laïcs, travaillant dans ses départements et espaces de formation: Gustavo Gutiérrez, Juan Luis Segundo, Segundo Galilea, Joseph Comblin, Juan Carlos Scannone, Lucio Gera et, comme seul laïc, Enrique Dussel. Les figures religieuses et intellectuelles qui s'imposent contrôlent nécessairement des espaces nationaux de réflexion et 
de formation. Pour le cas mexicain, il s'agit notamment du CIDOC (Centro Intercultural de Documentación), où, sous la protection de Sergio Méndez Arceo, le prêtre autrichien Ivan Illich et son équipe préparaient les missionnaires à la "réalité " latino-américaine. Il s'agit aussi du Secretariado Social Mexicano, chargé des œuvres sociales de la conférence nationale depuis 1929. À la fin des années 1960, il se situe à l'avant-garde du mouvement de rénovation pastorale et de l'intégration des sciences humaines à la réflexion chrétienne. Prenant ses distances avec la doctrine sociale de l'Église, cette réflexion développe une vision radicalisée du changement social.

Pour cette raison, le Secretariado Social Mexicano et le CIDOC, comme beaucoup d'autres organisations de la région, entrent en conflit avec l'autorité ecclésiale. Ils prennent leur autonomie entre la fin des années 1960 et le milieu des années 1970. La répression contre les thèses et acteurs de la théologie de la libération se généralise alors progressivement au sein de l'Église catholique et la déplace vers des espaces civils et œcuméniques de réflexion. Malgré tout, elle résiste au sein de la conférence épiscopale brésilienne et d'espaces religieux d'activisme intellectuel, en particulier jésuites.

\section{Convergences et intérêts de l'œcuménisme social}

Le contrôle interne du clergé sur les espaces ecclésiaux est réel, malgré les ouvertures vers les laïcs ${ }^{5}$. Son influence prépondérante s'exerce au-delà de ces espaces. Ainsi, si l'on établit une liste des principales organisations œcuméniques de la région, on observe le contrôle important exercé sur elles par les catholiques, en particulier clercs et ex-clercs. Or ces organisations sont entendues comme des espaces de décentrement militant et intellectuel vis-à-vis de l'Église catholique. Il y a certes un déplacement de perspectives, souligné par quelques (rares) abandons de charges cléricales, mais pas véritablement de rupture. Ses agents peuvent

5. L'établissement de corpus de théologiens de la libération et d'acteurs religieux et/ou intellectuels du champ confirme cette dominante cléricale. Nous avons d'abord construit un corpus de quarante-deux théologiens liés aux idées de la théologie de la libération, sur la base de critères, subjectifs, de reconnaissance et, objectifs, de présence dans ses réseaux entre la fin des années 1960 et le début des années 1990. Ils sont bien sûr majoritairement catholiques, quoique nous ayons pris en compte huit protestants. La présence religieuse est importante, couvrant la moitié du corpus, avec, en particulier, un quart de jésuites $(26,19 \%)$ et un autre quart $(26,19 \%)$ constitué de prêtres diocésains, non affiliés à un ordre religieux. Nous n'avons compté que deux laïcs, dont l'un, Enrique Dussel, n'était parfois considéré que comme un théologien. Il faut aussi tenir compte des quelques sorties du clergé, comme par exemple celles d'Hugo Assmann et, très tardivement, de Leonardo Boff. À ces quarante-deux théologiens, nous avons ajouté un corpus représentatif d'acteurs intellectuels et/ou religieux positionnés dans le champ, bien que peu souvent reconnus comme théologiens au sens strict du terme. Nous y avons distingué des cercles selon leur visibilité et position plus ou moins centrale. Les pourcentages favorisent davantage les laïcs (un peu plus de $20 \%$ ), mais dans la mesure où, en dehors de quelques cas exceptionnels, ils appartiennent à des cercles d'acteurs, certes transnationalisés, mais à la visibilité plus locale. 
continuer à développer leurs activités, aux marges de l'institution. Ils y travaillent avec des acteurs non catholiques, bénéficiant de l'amplitude de leurs solidarités militantes. Par ailleurs, ils continuent à collaborer avec les réseaux religieux de l'Église catholique, en particulier quand un évêque leur ouvre les portes de son diocèse. Ils tirent un profit symbolique, social et économique de la conservation de puissants relais ecclésiaux et militants dans le monde catholique. Même aux marges de l'institution, les conditions de promotion religieuse et intellectuelle dépendent du positionnement dans les dynamiques transnationalisées de ces réseaux ecclésiaux et d'ONG. Le contrôle d'espaces d'activisme intellectuel et d'animation militante s'étend au-delà des seuls clercs catholiques. Cependant, la nature bureaucratique de la construction de capital religieux et intellectuel investit, sur le plan militant, des capitaux sociaux et symboliques qui la lient intimement à des positions et relais ecclésiaux.

Le CEE (Centro de Estudios Ecuménicos) de Mexico est fondé par un pasteur luthérien, Rolf Lahussen, en 1968, mais le virage décisif de ce centre vers la définition militante d'un « œuménisme social » se produit en 1974. Cet œcuménisme prétendant dépasser les objectifs initiaux du dialogue interconfessionnel, il veut rendre compte des engagements communs de catholiques et de protestants au sein des luttes sociopolitiques de l'Amérique latine. Celles-ci aiguisent les divisions internes aux confessions entre les alliés des "pauvres » et ceux du statu quo social. Le CEE est alors passé sous la direction du prêtre-ouvrier catholique et missionnaire hollandais Franz Vanderhoff. L'Instituto Bartolomé de las Casas de Lima est fondé, la même année, par le prêtre péruvien Gustavo Gutiérrez, souvent considéré comme le "père " de la théologie de la libération.

Hugo Assmann, ex-prêtre et l'un des pionniers de la théologie de la libération, initie le projet du DEI (Departamento Ecuménico de Investigaciones) de San José, au Costa Rica, en 1976, et le dirige jusqu'en 1981 ; direction reprise ensuite par deux protestants (José Duque et Carmelo Álvarez), puis par un laïc catholique allemand (Franz Hinkelammert), avant que le théologien chilien Pablo Richard, un autre clerc catholique, en prenne la charge en 1998. Cette alliance entre catholiques et protestants se contruit autour de deux pôles interdépendants : le DEI et le SBL (Seminario Bíblico Latinoamericano). Au sein de cette institution théologique, le couple José Duque-Elsa Tamez bénéficie du capital symbolique de leur connexion avec le DEI. Pour sa part, le DEI tire des bénéfices financiers incontestables de cette alliance : ses cinq principaux bailleurs de fonds étant des ONG protestantes de Hollande, d'Allemagne, de Suède et de Suisse. Les deux personnages prédominants du DEI, les catholiques Franz Hinkelammert et Pablo Richard, en sont les emblèmes et contrôlent respectivement le séminaire de chercheurs invités et le cours de formation sociothéologique et pastorale, donnés par le DEI à ses clientèles militantes externes. Ces dernières, même si elles débordent les frontières $\mathrm{du}$ monde catholique, sont principalement liées à des réseaux d'ONG catholiques. Les convergences idéologiques de l'œcuménisme social s'accompagnent donc d'intérêts partagés par ses acteurs. 
Autour du CESEP (Centro Ecumênico de Serviços à Evangelização e Educação Popular), les mêmes intérêts réunissent catholiques et protestants. Tandis qu'un prêtre brésilien, José Oscar Beozzo en est le directeur, le méthodiste uruguayen Julio de Santa Ana en devient le sous-directeur en 1985. L'arrivée au Brésil de ce dernier fait suite à un exil suisse initié en 1974 et qu'il rompt provisoirement en devenant missionnaire des Églises protestantes suisses de langue française à Sao Paulo. Le CESEP consolide ses liens avec le Conseil Ecuménique de Genève, dont Julio de Santa Ana, devenu un représentant influent et qui prend la nationalité suisse.

Le CAM (Centro Antonio Montesinos) de Mexico est le produit, en 1979, d'une initiative commune de clercs et laïcs catholiques. Ils prétendent intervenir dans les débats de la conférence épiscopale latino-américaine de Puebla et informer l'opinion publique sur leurs évolutions. Cette stratégie de pénétration de la conférence fait suite à l'exclusion des " conseillers » liés aux idées de la théologie de la libération de la CELAM. Elle s'organise dans des commissions créées aux marges de la conférence avec la complicité des évêques "amis ", en majorité brésiliens. Ceux-ci rendent compte du contenu des discussions et y font entendre la voix des conseillers marginalisés. Le CAM est l'un des acteurs mexicains de la résistance à la nouvelle direction de la CELAM et compte à l'extérieur avec le soutien d'autres organisations œcuméniques de la région, et, dans l'Église catholique, avec des complicités religieuses et sacerdotales. La figure de la résistance des réseaux de la théologie de la libération, depuis le Mexique, est l'évêque Sergio Méndez Arceo qui s'associe à l'initiative internationale de Sergio Torres, ex-membre des Chrétiens pour le Socialisme au Chili et exilé en Amérique du Nord, qui organise une réunion avec le soutien de l'ordre religieux des Maryknolls à New York, en 1978. La maison d'édition Orbis Books, liée à cet ordre religieux, diffuse les textes de la théologie de la libération en langue anglaise. Cet ordre est un soutien important, notamment financier, pour la révolution sandiniste et ses réseaux catholiques au Nicaragua. La connexion du CAM avec le monde ecclésial et ses enjeux ne s'en accompagne pas moins de tensions autour de l'axe théologique et "scientifique " de sa réflexion et de son action. Sans cesser de participer de préoccupations et de réseaux liés au monde catholique, il s'oriente, en effet, vers une conception plus laïque de sa mission sociale. Au début des années 1990, les tensions internes conduisent au divorce entre le groupe théologique " historique » de l'organisation, qui doit être refondé, et son groupe de sciences sociales.

Ces mêmes liens transnationaux impliquent d'autres organisations œcuméniques, généralement dirigées par des clercs catholiques : le CAV (Centro Antonio Valdivieso) de Managua, où régne le prêtre catholique Uriel Molina ; le CEBI (Centro de Estudios Bíblicos) fondé dans la banlieue de Rio de Janeiro, en 1979, par le missionnaire et carmélite hollandais Karl Mesters; le Centro Diego de Medellín de Santiago du Chili et IDEAS (Instituto de Estudios y Acción Social) de Buenos Aires. 


\section{Le rapport ambivalent au pouvoir ecclésial}

On pourrait objecter que ces acteurs, clercs ou non, firent le choix du décentrement de l'Église catholique vers le « monde » et de la théologie vers les sciences sociales. Leur option politique, engagée dans des solidarités militantes qui débordent le monde catholique, leur vaut de violents conflits avec l'autorité ecclésiale. On pourrait également souligner le poids des laïcs dans ces solidarités militantes, au-delà de leur anonymat et de la visibilité individuelle des théologiens et des clercs qui s'associent à eux.

Mais le problème posé concerne également la réalité ambivalente du rapport généralisé du champ au pouvoir ecclésial. Autour de la théologie de la libération, on n'assiste pas véritablement à une redistribution de ce pouvoir. Au contraire, comme nous l'avons déjà souligné, les grandes figures cléricales de la théologie de la libération s'en trouvent consolidées. Le service revendiqué à la communauté et la définition de rapports plus égalitaires avec les laïcs n'impliquent pas un changement de nature de leur autorité : il en renforce la légitimité. Le déplacement de clercs vers les marges œcuméniques et civiles de l'activisme intellectuel et social de la théologie de la libération implique une recomposition alternative de ce pouvoir qui se réalise aux frontières de l'usage symbolique de la "périphérie », dont participe la théologie de la libération, et du "centre », dont on mobilise les ressources institutionnelles, sociales et économiques. Le " centre ", même débordé, ne cesse pas de réguler les dynamiques qui le décentrent.

On peut donc mieux comprendre l'importance que prennent les réseaux religieux dans la diffusion des idées de la théologie de la libération. D’une part, le réseau religieux définit un type transversal de solidarités, situées dans l'espace transnational. À l'instar des réseaux militants dont il participe, il contourne et défie ainsi la structure hiérarchique et diocésaine de pouvoir de l'Église catholique, établie aux niveaux national et local. D'autre part, le religieux n'en constitue pas moins une figure cléricale. Il se situe exactement au carrefour des dynamiques sociales qui défient le pouvoir ecclésial et de la recomposition déplacée de ce dernier. La valeur " anti-institutionnelle » de la théologie de la libération, même si elle n'est pas dépourvue d'effets sociopolitiques (pas seulement au Brésil), provient moins de sa dimension locale de «mouvement social " que de sa dimension transnationale de "réseau ».

La manifestation la plus évidente de la régulation cléricale du champ est l'apparition, simultanée à celle de la théologie de la libération, de mouvements de prêtres. Ils rendent témoignage de la participation sacerdotale à la radicalisation sociopolitique des années 1960, tout en prétendant en réguler la signification pour l'ensemble des chrétiens engagés. C'est le cas, en 1968, du mouvement ONIS (Oficina Nacional de Información Sacerdotal) au Pérou, des Prêtres pour le Tiers-monde en Argentine et du groupe Golconda en Colombie. Suivent de nombreux autres, dont en particulier le Groupe des 80 au Chili (lequel débouche 
en 1971 sur le mouvement des Chrétiens pour le Socialisme) et les Prêtres pour le Peuple, au Mexique, en 1972. La conférence donnée par Gustavo Gutiérrez à Chimbote en 1968 est considérée comme le premier usage public attesté de l'expression "théologie de la libération » dans un contexte catholique (Gutiérrez, 1969). S'y croisent des militants de l'UNEC (Unión Nacional de Estudiantes Catolicos), mouvement de la MIEC-JECI dont Gutiérrez est aumônier, et les prêtres du mouvement ONIS.

Autour des Chrétiens pour le Socialisme, on trouve les mêmes convergences entre les prêtres du Groupe des 80 et le militantisme laïque. Comme nous l'expliqua Pablo Richard, les prêtres y jouent un véritable rôle de direction de conscience qui consiste à assurer que les chrétiens soient "totalement révolutionnaires » sans renoncer à être « totalement chrétiens ". Il faut anticiper le possible désarroi des militants chrétiens qui peuvent être tentés de sacrifier leur identité chrétienne à leurs engagements politiques. La conciliation recherchée entre les valeurs évangéliques et l'option révolutionnaire est un enjeu particulier du champ. Elle justifie la médiation sacerdotale entre l'Église, qui ouvre des espaces à l'activisme laïque, et les dynamiques sociopolitiques, auxquelles s'intègrent les prêtres. Ce double mouvement d'intégration militante et de régulation institutionnelle distingue la théologie de la libération d'autres formes de radicalisation de secteurs catholiques dans la région, notamment moins préoccupés pour fixer la nature de la relation entre foi et politique : par exemple la fondation de l'ELN en Colombie par le prêtre Manuel Pérez, lequel, dans le même contexte de radicalisation, prend directement la voie de la lutte armée.

\section{L'hypothèse intransigeante et l'impossible rupture religieuse}

À l'intérieur de l'Unité Populaire, la plupart des prêtres du Groupe des 80, comme Gonzalo Arroyo et Sergio Torres, sont surtout proches du MAPU (Movimiento de Acción Popular Unitaria), produit de la scission interne de la Démocratie Chrétienne en 1969. Une minorité d'entre eux, dont Pablo Richard, optent pour d'autres groupes politiques, comme le MIR (Movimiento de Izquierda Revolucionaria), guérilla urbaine d'inspiration castriste, où la plupart des laïcs catholiques militaient.

L'intégration à l'Unité Populaire et ce pluralisme politique marquent une différence certaine avec le projet de la Démocratie Chrétienne. Celle-ci prétend à la fois déconfessionnaliser l'action politique et la fonder, dans le «monde ", sur les valeurs chrétiennes (Compagnon, 2003) ${ }^{6}$. Dans l'optique de la théologie de la libération, le choix d'un parti répond à une détermination stratégique plus

6. Olivier Compagnon, qui s'intéressa à l'impact de la pensée de Jacques Maritain en Amérique du Sud, souligne la contradiction entre le discours de déconfessionnalisation et la dominante confessionnelle de la Démocratie Chrétienne. 
radicalement autonome face au point de vue de la foi religieuse. Elle s'écarte ainsi de l'idéologie de la troisième voie, une voie proprement chrétienne de société, qualitativement supérieure aux voies libérale et socialiste. Or, Émile Poulat fait de la conscience de l'originalité de cette voie le signe distinctif du catholicisme intransigeant, comme type historique de catholicisme qui avait prédominé au sein du catholicisme contemporain. Il le définit comme un catholicisme d'opposition à la modernité, dont la tendance majoritaire opte ensuite pour l'adaptation. Cette dernière n'a pas pour autant renoncé à infléchir les valeurs modernes de progrès et d'émancipation dans le sens de son refus initial (Poulat, 1977 : 198).

Néanmoins, au sein de la théologie de la libération, l'abandon de l'idée de troisième voie ne signifie pas forcément une rupture avec ce type de catholicisme. Elle projette l'ambition d'approfondir la signification chrétienne des engagements pris sur la notion d'un socialisme proprement latino-américain. Les expériences latino-américaines de "libération » sont en effet considérées irréductibles aux modèles de la modernité capitaliste et socialiste, liés à la sécularisation européenne. Le mouvement apparent vers un catholicisme plus " moderne », " progressiste » et " sécularisé » s'accompagne de la défense des contributions chrétiennes, fondamentalement catholiques, à une vision latino-américaine de la libération politique. Foi et politique, sans se confondre totalement, sont entendues comme inséparables. Cela se reflète dans l'exigence de compromission politique de l'institution et de ses cadres, dans le rejet de la privatisation de la foi et dans l'évocation de la dimension "communautaire " et "intégrale" de la libération souhaitée. En même temps qu'on se solidarise avec le projet émancipateur de la révolution sociale, on cherche à mobiliser les solidarités, valeurs et religiosité traditionnelles $\mathrm{du}$ «peuple».

Cette ambiguïté fait de la théologie de la libération une forme de différenciation interne du catholicisme intransigeant qui se redéploie depuis l'Europe à travers les réseaux du tiers-mondisme catholique. L'impossibilité de la rupture est confirmée sur le plan du rapport des acteurs, cléricaux et décentrés, au pouvoir ecclésial. Agents et victimes du pouvoir ecclésial, dont ils imposent et acceptent la régulation, les catholiques en restent dépendants. Le symbole de cette dépendance est le prêtre et ministre de la culture du gouvernement sandiniste au Nicaragua, Ernesto Cardenal. Lors de la visite du pape Jean-Paul II, en 1983, il se laisse réprimander par lui, à genoux, en présence des médias internationaux, après avoir désobéi à son ordre de quitter le gouvernement. Il faut souligner, en ce sens, la rareté des sorties du clergé : lorsqu'elles se produisent, c'est après un long combat pour demeurer au sein de l'institution. Leonardo Boff quitte définitivement le clergé et l'ordre des franciscains en 1992, après s'être d'abord plié au jugement de la congrégation pour la doctrine de la foi en 1984. On y revient parfois comme, par exemple, Pablo Richard. Marié avec une ex-activiste du MIR, lors de son exil en France en 1974, puis divorcé, il récupère son statut de prêtre à son retour en Amérique latine, au Costa Rica en 1978. On ne s’éloigne 
de toute façon jamais beaucoup, rebondissant dans les réseaux d'ONG et de militants chrétiens du champ.

Cette obstination à demeurer n'est aucunement désintéressée : le conflit ecclésial, dont la polémique fait rage dans les années 1980, oppose deux visions de l'institution et de sa mission. Cependant, les uns et les autres tentent d'éviter, à tout prix, la rupture. L'option préférentielle pour les pauvres de la conférence épiscopale latino-américaine de Puebla (CELAM, 1984) ${ }^{7}$, interprétable de façon très contrastée, est le produit de cette double logique de confrontation et de recherche de compromis. On préfére régler les «affaires » en famille.

\section{L'imaginaire de la périphérie}

Tout cela oblige à tirer des conclusions sensiblement différentes de celles de M. Löwy et à rejeter la thèse d'un mouvement univoque de "conversion " de l'Église catholique à la problématique du changement social de sa périphérie vers son centre. On observe plutôt un mouvement de balance entre la périphérie et le centre de l'institution, constitutif des ambivalences des intérêts sociaux du champ. Cela explique le double mouvement contradictoire observé de régulation cléricale et de généralisation militante. À partir du moment où l'on se dégage de l'idée d'un vaste mouvement social, dont la théologie de la libération aurait été l'expression, la nature même de cette " périphérie » fait problème. Dans le discours théologique de la libération, la conscience "périphérique » implique le rejet de la domination du « centre » et la nécessité de la « praxis » qui doit le guider. Il s'y fait jour une fascination incontestable pour la figure de l'intellectuel " organique ", articulé au mouvement populaire de libération. Pourtant, cette intégration est plus fictive que réelle et témoigne plutôt d'un certain usage intellectuel du vocable de la "praxis ", exploitant le capital symbolique de son positionnement social au carrefour du centre et de la périphérie.

Le champ articule des activités religieuses, pastorales, académiques et militantes. Toutefois, le type d'activités qui lui est le plus propre ne se rapporte à aucun de ces plans. Si l'on reprend les corpus des acteurs prédominants dans les conditions de la visibilité de la théologie de la libération, on observe l'inégalité de leurs investissements directs dans l'action pastorale, sociale et politique. On note aussi la distance de certains d'entre eux avec l'activité proprement religieuse. On souligne enfin le développement de leur réflexion intellectuelle aux marges du champ académique. Comme on l'a déjà avancé, celle-ci se concentre principalement dans les espaces parallèles, ecclésiaux puis civils et œcuméniques d'activisme intellectuel du champ. Pour la plupart, les théologiens de la libération sont professeurs dans les facultés de théologie des universités catholiques et dans les

7. Le document officiel, approuvé par Rome, fut publié en 1984. Dès 1979, une version non encore approuvée circula en langue espagnole. 
séminaires de formation, mais ils se déplacent progressivement vers ces espaces parallèles. À l'intérieur du champ des sciences humaines, leur position de théologiens est à plus forte raison périphérique et de second plan. Pourtant, le poids moral de leurs engagements leur permet d'y gagner des audiences importantes, depuis l'Amérique latine vers l'Europe et l'Amérique du Nord, et d'y jouir d'un effet certain de promotion.

C'est précisément leur activisme intellectuel, développé aux marges des champs religieux et académique, qui constitue le plan d'activités le plus propre aux réseaux de la théologie de la libération. Son axe militant de développement est la condition nécessaire de sa valeur de rénovation religieuse et théologique, comme celle de sa valeur d'alternative intellectuelle. Il intègre le point de vue des sciences humaines sur le terrain revendiqué de l'action collective, où il investit l'originalité de ses visions et enjeux religieux et théologiques. La position périphérique de ses agents dans le champ académique s'y convertit en un atout symbolique pour la revendication de l'originalité de leurs expériences sociales.

\section{Les usages intellectuels de la " praxis "}

Le contexte militant de production des premiers textes catholiques de la théologie de la libération favorise la reconnaissance de leur rôle fondateur (Gutiérrez, 1969 ; Assmann, 1971) ; textes principalement publiés par le secrétariat latinoaméricain de la MIEC-JECI (Mouvement international des étudiants catholiques - Jeunesse étudiante catholique internationale) et les éditions Tierra Nueva du mouvement protestant ISAL (Iglesia y Sociedad en América Latina) à Montevideo. En revanche, la légitimité fondatrice de la thèse antérieurement soutenue du protestant Rubem Alves est de plus en plus discutée (Alves, 1968) à cause de ses développements peu orthodoxes, du point de vue du champ : la référence à Nietzsche l'emporte sur celle à Marx. La reprise de la critique marcusienne des sociétés industrielles avancées éclipse l'urgence de la dénonciation de la situation de dépendance et de misère sociale de l'Amérique latine, ce qui est dû aussi à son contexte académique de production, écrite en anglais, aux États-Unis, au séminaire de Princeton.

Si l'on se prend à interroger la faiblesse intellectuelle de la théologie de la libération, on s'entend répondre que sa richesse se situe ailleurs : dans la profondeur de ses engagements et dans son intériorisation du " cri des pauvres ». D'abord, on fait la distinction entre le caractère concret et engagé de la théologie de la libération et l'abstraction de la pensée européenne, purement idéale. On en fait aussitôt une autre entre la transmission du savoir spécialisé, nécessaire à la « praxis ", et son investissement immédiat dans l'action au niveau des « bases ». Sur le plan religieux, les communautés ecclésiales de base, censées subvertir la 
structure ecclésiale, lui sont subordonnées. De la même façon, sur le plan intellectuel, la subversion militante de la régularité normative du savoir est assujettie aux règles de la différenciation sociale entre le «savant » et le «commun ».

Cette double justification occulte une double inconsistance : celle de la qualité de la réflexion intellectuelle menée et celle de la profondeur réelle des engagements qui la légitiment. Elle autorise paradoxalement la production d'une vaste littérature grise, supposée ne pas être la finalité réelle de cet activisme intellectuel, en dehors de tout critère académique de validation scientifique. Les centres œcuméniques cités, relayés par les éditeurs catholiques, deviennent ainsi des centres importants d'édition, grâce à l'argent des ONG internationales. Le caractère répétitif et rigide de cette production intellectuelle est manifeste. Le recours magique à la notion de "praxis » justifie tout. La critique de l'observateur extérieur, pas en mesure de comprendre la profondeur "pratique » de ses enjeux sociaux et éthiques, est immédiatement censurée. Toutefois, cette censure protége moins la réalité de l'articulation avec un sujet populaire de transformation sociale que la critique possible de la faiblesse de cette articulation. Les ressources économiques et les capitaux sociaux mobilisés au sein des réseaux religieux et militants du champ sont nécessaires à sa reproduction. Inversement, la référence aux expériences "vécues » de l'Amérique latine permet d'y mobiliser ces ressources. Un autre aspect frappant est l'intensité de la circulation internationale des acteurs de ces réseaux, entre l'Europe, l'Amérique du Nord et l'Amérique latine, qui marque précisément la distance entre les expériences locales, au niveau desquelles on revendique les engagements pris, et les solidarités transnationales qui permettent de les capitaliser.

\section{Les paradoxes de l'évocation populaire}

Ces réseaux prennent leur origine dans les tiers-mondismes catholique et protestant des années 1940 à 1950 et 1960, articulés autour du thème du développement. Les activités intellectuelles orientées vers l'action sociale et pastorale s'y consolident : par exemple, les CIAS (Centro de Investigación y Acción Social), fondés par les jésuites dans les capitales et grands centres urbains de la région; les multiples espaces de réflexion sociale et de formation pastorale qui dépendent des conférences épiscopales nationales et de la CELAM. Le DEI de San José développe, par exemple, les mêmes activités que l'ILADES (Insituto Latinoamericano de Doctrina y Estudios Sociales), fondé à Santiago du Chili en 1966. Le premier est conçu par Hugo Assmann comme un espace de formation et de réflexion pour les chrétiens engagés dans les « luttes de libération " de la région. Le second est créé afin de mettre en pratique la doctrine sociale de l'Église. Le 31 décembre 1969, les chercheurs « radicalisés » qui ont rompu avec la Démocratie Chrétienne en faveur de l'Unité Populaire en sont exclus. Franz Hinkelammert, future figure du DEI, fait parti des exclus : il reconnaît lui-même la continuité 
du type d'activité intellectuelle liant les deux organisations. En Colombie, le CINEP (Centro de Investigación y Educación Popular), produit, en 1976, d'une scission interne du CIAS de Bogota, cette fois au bénéfice des plus radicaux, est un autre exemple de réappropriation, autour du thème de la libération, d'activités intellectuelles initiées dans le contexte développementiste du tiersmondisme catholique. Son contenu sociopolitique se radicalise, ainsi que sa conception engagée de l'intellectuel "organique" au service du mouvement populaire et l'intégration revendiquée du point de vue de la foi aux objectifs politiques de la révolution sociale.

Les conflits et discontinuités, qui s'y imposent, manifestent une corrélation entre la radicalisation politique des années 1960, en Amérique latine, et des secteurs de l'Église catholique. Mais l'articulation dynamique entre la théologie de la libération et les objectifs de changement social. L'articulation entre la radicalisation révolutionnaire des années 1960 et l'émergence d'un sujet populaire ne va pas de soi. Il ne s'agit pas, bien sûr, d'ignorer la réalité de la violence sociale et de la pression populaire impliquées dans cette radicalisation. Néanmoins, ses analyses sociologiques ont aussi montré le poids idéologique considérable de classes moyennes urbaines et intellectualisées, notamment issues des universités qui abusent de la référence populaire (Touraine, 1988) ${ }^{8}$. Dans l'orbite de la théologie de la libération, ce rapport d'alibi, ou du moins paradoxal, avec le sujet populaire invoqué reflète l'ambivalence de la position systématiquement intermédiaire du champ. La sensibilité révolutionnaire de ses agents témoigne de leur proximité sociologique avec les classes moyennes radicalisées. Ils interagissent avec elles et partagent, dans de nombreux cas, leurs origines et leurs expériences sociales ${ }^{9}$. On comprend mieux, dès lors, les dynamiques contradictoires du champ entre la formation d'un pouvoir religieux " alternatif ", débordé par ses dynamiques militantes, et celle d'un type d'intellectuel chrétien, inégalement investi dans ces dynamiques. Ces deux plans peuvent se superposer ou se contredire. Dans tous les cas, la tension qui les organise, constitutive de leurs intérêts sociaux communs, explique aussi leur interdépendance.

8. Une analyse "désidéologisée ", conduite en terme de mouvement social, peut ainsi être démystifiante. Notre approche n'en est pas forcément exclusive et peut en combiner les éléments à la singularité de l'éclairage qu'elle apporte.

9. Les acteurs résistent à donner des détails sur leurs origines sociales ou s'inventent une mythologie personnelle, peu concordante avec leur réalité sociologique. Malgré tout, nous avons pu réunir suffisamment d'informations pour observer qu'ils sont, en majorité, issus de classes moyennes urbaines et provinciales, voire, pour les latino-américains, de migrations européennes récentes en Amérique latine. Leur origine sociale n'est pas l'objet central de l'analyse du rapport paradoxal entre la promotion d'un type intellectuel et la référence populaire, mais elle en renforce la pertinence. 


\section{Des réseaux transnationaux}

De ce point de vue, l'aspect le plus controversé du discours de justification de la théologie de la libération est son identification univoque avec la réalité latino-américaine. Les éléments qui permettent d'envisager la nature transcontinentale et transnationale de cette dynamique sont multiples : sa généalogie exogène dans le tiers-mondisme catholique et protestant; la présence importante de missionnaires européens et nord-américains dans le champ; l'intensité de la circulation internationale des acteurs ; leurs études dans des universités et séminaires représentatifs de la rénovation de la pensée chrétienne en Europe ; le rôle initial joué par la CELAM, les ordres religieux, représentés dans la CLAR (Conferencia Latinoamericana de Religiosos), et les mouvements laïques internationaux, en particulier de la MIEC-JECI; la multiplication postérieure des réseaux de solidarité internationale et les soutiens financiers et moraux qui en résultent ; la régularité des rencontres théologiques internationales; l'étroitesse des échanges ntellectuels et éditoriaux. Ces réseaux interagissent avec les contextes locaux et nationaux, où ils se focalisent selon les circonstances ecclésiales, sociales et politiques.

Il existe une contradiction entre le discours d'opposition symétrique entre la "périphérie» dominée et le «centre» et, par ailleurs, cette proximité sociologique du "centre » au sein des conditions réelles de construction de l'imaginaire de la «périphérie ». De ce point de vue, le rapport de ces réseaux aux réalités locales et nationales n'est jamais transparent : il est donc vain de vouloir chercher directement dans le contexte de pauvreté du Brésil la raison d'être de la théologie de la libération. Il est également vain de chercher actuellement, dans la problématique indigène du Chiapas, la raison d'être du projet d'une théologie indigène. Malgré la réalité de la misère sociale et les effets incontestables de mobilisation sociale de ces discours, la mise en regard de la pauvreté et de la réalité indigène est médiatisée par les intérêts et les visions propres à ces réseaux. Le "pauvre » et «l'indigène » sont, en grande partie, la construction imaginaire de ces réseaux et non un effet immédiat de la réalité. Le diocèse de San Cristobal de la Casas, avant de se redécouvrir comme un territoire indigène, est un lieu de passage des réseaux missionnaires, réseaux relayés par les ONG internationales, et directement impliqués dans le « réveil » local.

Latino-américaine, la théologie de la libération le fut, mais dans le sens de ces ambivalences. Ces dernières singularisent aujourd'hui encore l'axe théologique et l'environnement idéologique de l'ethos de transformation sociale des réseaux militants, religieux et intellectuels qui se situent dans sa trajectoire.

Malik Tahar CHAOUCH

Universidad Autónoma del Estado de Hidalgo, Mexique 
26 - Archives de sciences sociales des Religions

\section{Bibliographie}

Alves Rubem, Towards a Theology of Liberation, Princeton, University of Princeton Press, 1968.

Assmann Hugo, Liberación-opresión : desafío a los cristianos, Montevideo, Tierra Nueva, 1971. (Reprise amplifiée de Teología de la liberación. Una evaluación prospectiva, Montevideo, MIEC-JECI, 1970).

BERryman Phillipp, Liberation Theology. The essential Facts about the Revolutionary Movement in Latin America and Beyond, New York, Pantheon Books, 1987.

Bigo Pierre, Débat dans l'Église : théologie de la libération, Mareil-Marly, Aide à l'Église en Détresse, 1990.

Blancarte Roberto, Historia de la Iglesia católica en México, Mexico, Fondo de Cultura Económica, 1992.

Bourdieu Pierre, Questions de sociologie, Paris, Éditions de Minuit, 1984.

Bourdieu Pierre, Wacquant Loïc, Réponses. Pour une anthropologie réflexive, Paris, Éditions du Seuil, 1992.

CELAM, La Evangelización en el presente y en el futuro de América latina. III Conferencia General del Episcopado Latinoamericano, Mexico, Librería Parroquial de Claveria, 1984.

Cierva y DE Hoces Ricardo de la, La Hoz y la Cruz: ange y caída del marxismo y la teología de la liberación, historia reservada de los pontificados de Pablo VI, Juan Pablo I y Juan Pablo II sobre ocho mil documentos inéditos, Madrid, Fénix, 1996.

Colonomos Ariel, Églises en réseaux, Paris, Presses de Sciences Po, 2000.

Compagnon Olivier, Jacques Maritain et l'Amérique du Sud. Le modèle malgré lui, Lille, Septentrion, 2003.

Concha Miguel, "Teología de la liberación » in Noberto Matteucci Bobbio, Nicola Pasquino, Gianfranco Pasquino, eds., Diccionario de política, Mexico, Siglo XXI, 1997, pp. 1557-1563.

CORTEN André, Le pentecôtisme au Brésil. Émotion du pauvre et romantisme théologique, Paris, Karthala, 1995.

-, «Une mise en réseaux de la théologie de la libération » in Jean-Pierre BAstian, (dir.), Europe latine-Amérique latine : la modernité religieuse en perspective comparée, Paris, Karthala, 2001, pp. 267-285.

Del Valle Luís, «Teología de la liberación en América Latina », in Roberto Blancarte, (comp.), El pensamiento social de los católicos mexicanos, Mexico, Fondo de Cultura Económica, 1996, pp. 230-265.

Gutierrez Gustavo, Hacía una teología de la liberación, Montevideo, MIEC-JECI, 1969.

-, Teología de la liberación : perspectivas, Lima, CEP, 1971.

HewiTT Warren, BuRdick John, eds., The Church at the Grassroots in Latin America: Perspectives on Thirty Years of Activism, London, Praeger, 2000.

Jimenez Roberto, Tensiones y crisis en la Iglesia latinoamericana: desde Puebla hasta la Libertatis conscientia 1979-1987, Bogota, Centro de Estudios para el Desarrollo e Integración de América Latina, 1988.

LeHmann David, Struggle for the Spirit: Religious Transformation and Popular Culture in Brazil and Latin America, Cambridge, Polity Press, 1996.

LEPELEY Joaquín, Teología de la liberación : un análisis temático cronológico, Caracas, Arte, 1985. 
Levine Daniel, Mainwaring Scott, eds., Religion and Popular Protest in Latin America, Notre Dame, University of Notre Dame Press, 1986.

LeVIne Daniel, "L'impact du discours de la théologie de la libération en Amérique latine : les communautés ecclésiales de base ", Archives des Sciences Sociales des Religions, 71, Paris, juillet-septembre 1990, pp. 43-62. (Numéro spécial des Archives des Sciences Sociales des Religions consacré aux théologies de la libération en Amérique latine).

-, Popular Voices in Latin American Catholicism, Princeton, Princeton University Press, 1992.

Löwy Michael, La guerre des dieux. Religion et politique en Amérique latine, Paris, Éditions du Félin, 1998.

LyNCH Edward Anthony, Political-Religious Expressions: Liberation Theology and Christian Democracy in Latin America, Charlottesville, University of Virginia, 1990.

MaInwaring Scott, Wilde Alexander, eds., The Progressive Church in Latin America, Notre Dame, University of Notre Dame Press, 1988.

Mallimaci Fortunato, Catholicisme et État militaire en Argentine, 1930-1946, Paris, École des Hautes Études en Sciences Sociales, 1988.

MATAS, Juan, "Éléments pour une approche des diasporas de l'exil chilien en Europe et des réseaux religieux liés à la théologie de la libération » in Jean-Pierre BASTIAN, (dir.), Europe latine-Amérique latine : la modernité religieuse en perspective comparée, Paris, Karthala, 2001, pp. 257-265.

Meyer Jean, Samuel Ruiz en San Cristóbal, Mexico, Editorial Tusquets, 2000.

Muro GonzÁlez Víctor Gabriel, La teología de la liberación como expresión ideológica de cambio social en México, Mexico, Universidad Nacional Autónoma de México, 1982.

-, « La Iglesia ante la movilización social en el México contemporáneo : una perspectiva teórica » in Víctor Gabriel Muro González, Manuel Canto (coord.), El Estudio de los movimientos sociales : teoría y método, Mexico, El Colegio de Michoacán, UAMX, 1991, p. 155-174.

-, Iglesia y movimiento social en México, 1972-1987, los casos de ciudad Juárez y el Istmo de Tehuantepec, Zamora, Red Nacional de Investigación Urbana, 1994.

OLIVEIRos Roberto, Liberación y teología : génesis y crecimiento de una reflexión 19661976, Lima, CEP, 1977.

Poulat Émile, Église contre bourgeoisie. Introduction au devenir du catholicisme actuel, Tournai, Casterman, 1977.

SAUVAGE Pierre, "Relations entre Belges et Latino-américains à propos de la théologie de la libération (1970-1980) " in Jean-Pierre BASTIAN (dir.), Europe latine-Amérique latine: la modernité religieuse en perspective comparée, Paris, Karthala, 2001, pp. 287-306.

Silva Gotay Samuel, El pensamiento cristiano revolucionario en América Latina y el Caribe: implicaciones de la teología de la liberación para la sociología de las religiones, Salamanque, Sígueme, 1981.

SмIтH Christian, The Emergence of Liberation Theology. Radical Religion and Social Movement Theory, Chicago, University of Chicago, 1991. (Trad. espagnole : La teología de la liberación: radicalismo religioso y compromiso social, Buenos Aires, Paidós, 1994).

Touraine Alain, La parole et le sang. Politique et société en Amérique latine, Paris, Odile Jacob, 1988. 


\section{Résumé}

Cet article propose une nouvelle approche de la théologie de la libération en Amérique latine. À partir d'une lecture critique de la littérature sur le thème, nous prenons nos distances avec la notion indifférenciée d'un vaste mouvement social, au bénéfice de l'analyse d'un champ social. Nous reconstruisons ainsi les intérêts différenciés des acteurs intéressés par ses enjeux sociaux. Nous y mettons en évidence le rapport ambigu de ses agents catholiques au pouvoir ecclésial constitué et à son idéologie. Nous y dessinons les traits d'un type intellectuel qui entretenait un rapport pour le moins paradoxal avec le sujet populaire censé fonder sa réflexion. Enfin, nous y montrons le rôle central joué par les réseaux transcontinentaux et transnationaux du tiers-mondisme chrétien, qui invoquaient la réalité latino-américaine dans les termes d'une opposition simpliste entre la "périphérie» dominée et le "centre».

Mots-clés : théologie de la libération, christianisme contemporain, église catholique, Amérique latine, politique et religion.

\section{Abstract}

This article proposes a novel approach to the theology of the liberation in Latin America. From a critical reading of the literature on the subject, we moved away of the notion of an ample social movement, in benefit of the analysis of the social field. We reconstructed therefore the interests differentiated from the actors implied with its development. We put in evidence the ambiguous relation of its catholic agents with the constituted ecclesial power and its ideology. We drew the characteristics of a type of intellectual who maintained a paradoxical relation with the popular subject supposed to base his reflection. We finally showed the main role played by the transcontinental and transnational networks of the Christian third word that invoked the Latin American reality in the terms of a simple opposition between the dominated "periphery" and the "center".

Key words: theology of liberation, to-day christianity, catholic church, Latin America, politics and religion.

\section{Resumen}

Este artículo propone un acercamiento novedoso a la teología de la liberación en América latina. À partir de una lectura crítica de la literatura sobre el tema, nos alejamos de la noción indiferenciada de un amplio movimiento social, en beneficio del análisis de un campo social. Reconstruimos así los intereses diferenciados de los actores implicados con su desarrollo. Ponemos en evidencia la relación ambigua de sus agentes católicos con el poder eclesial constituido y con su ideología. Dibujamos los rasgos de un tipo de intelectual que mantenía una relación por lo menos paradójica con el sujeto popular que se suponía fundamentaba su reflexión. Mostramos finalmente el papel central jugado por las redes transcontinentales y transnacionales del tercermundismo cristiano que invocaban la realidad latinoamericana en los términos de una oposición simplista entre la "periferia" dominada y el "centro".

Palabras clave : teología de la liberación, cristianismo contemporáneo, America Latina, politica y religion. 


\section{Gwendoline Malogne-Fer}

\section{L'accès des femmes au pastorat Le cas de l'Église évangélique de Polynésie française}

En Polynésie française, l'appartenance au christianisme joue un rôle essentiel dans la définition des identités individuelles mais aussi de l'identité culturelle dite « traditionnelle ». Malgré un relatif affaiblissement des pratiques institutionnalisées, notamment la participation aux cultes, et la diversification en milieu urbain de l'offre religieuse, le protestantisme demeure une composante essentielle de cette identité culturelle traditionnelle qui ne s'acquiert pas par conversion mais par héritage. Ceux qui, à l'âge adulte, se convertissent à une nouvelle religion sont violemment accusés de manquer de respect à l'égard de leurs parents et de leurs ancêtres (Fer, 2005 : 344-351).

Les dernières estimations disponibles révèlent, à côté des deux grandes Églises " historiques ", protestante et catholique, qui continuent à rassembler près de $80 \%$ de la population en 1992, l'essor relatif de nouveaux groupes religieux (mormons, adventistes, pentecôtistes, témoins de Jéhovah et baha'i). L'Église évangélique de Polynésie française rassemble encore $44 \%$ de la population mais son taux de croissance "la place dans un processus de déclin continuel » (Ernst, 1997 : 83-88).

L'évangélisation initiée à la fin du XVIII ${ }^{e}$ siècle par des missionnaires britanniques de la London Missionary Society a associé le protestantisme à la « religion des Anglais » contre le catholicisme présenté comme la "religion des Français ». Cette configuration historique a favorisé au sein de l'Église évangélique de Polynésie française (EEPF) - rebaptisée Église protestante mâ'ohi en 2004 - une compréhension de l'authenticité polynésienne diamétralement opposée à la présence française sur le territoire polynésien. Le renouveau théologique, initié par la commission d'animation théologique depuis 1988, prône la libération du peuple mâ'ohi, le retour à la terre et à la langue mâ'ohi comme preuve d'une authentique foi protestante (Saura, 1989 ; Fer et Malogne-Fer, 2002). C'est dans ce contexte de déclin relatif du protestantisme et de renouveau théologique que la direction de l'Église évangélique de Polynésie française décide, en 1995, après plusieurs années de discussions, d'autoriser les femmes à accéder au pastorat. 
Mais auparavant, les femmes étaient déjà fortement impliquées dans le ministère en tant qu'épouses ${ }^{1}$.

De fait, l'officialisation des responsabilités des femmes en paroisse a suivi deux dynamiques distinctes : une institutionnalisation des activités exclusivement féminines et un égal accès aux fonctions pastorales et diaconales. Ce double processus qui repose sur une logique à la fois différentialiste et égalitariste crée des tensions entre une tendance autonomiste (les femmes entre elles) et une tendance intégrationniste (les femmes au côté des hommes) qui se retrouvent au niveau de l'institution ecclésiale et du couple pastoral ou diaconal. Le fil conducteur de cette réflexion, issue d'un travail de thèse ${ }^{2}$, consiste à interroger la transformation de l'institution ecclésiale et des modes d'exercice des ministères en lien avec la reconfiguration des relations entre les hommes et les femmes en paroisse.

La féminisation du corps pastoral transforme les représentations et les formes d'autorité au sein de la paroisse, elle questionne les pasteurs et les diacres sur les modalités d'exercice des ministères et les paroissiens sur les relations qu'ils entretiennent avec leur pasteur. Mais l'accession des femmes au pastorat entraîne également de nouvelles formes de relations conjugales et pose la question de la place du mari dans la paroisse et au sein du couple dans la mesure où l'Église refuse de consacrer les deux conjoints d'un même couple.

\section{Le couple pastoral}

L'Église évangélique de Polynésie française, protestante de tendance réformée, reconnaît officiellement trois ministères: le pasteur consacré (fa'atâhinu), le diacre installé ( ha'amau) et l'évangéliste envoyé (tono). Idéalement, le pasteur n'est pas uniquement chargé du côté spirituel mais aussi temporel et matériel de la paroisse, pour autant que cette distinction ait un sens. Marié et respecté, c'est un homme d'autorité qui ne doit pas abuser de cette autorité auprès des paroissiens.

Si les Réformateurs, au XVI ${ }^{\mathrm{e}}$ siècle, ont aboli l'obligation de célibat des prêtres, en soulignant la contradiction qu'il y avait à considérer le mariage comme un sacrement tout en le refusant à une catégorie particulière de personnes, ils n'ont pas rendu le mariage des pasteurs obligatoire (Reymond, 1991 : 14-15). Pourtant, en Polynésie française le pasteur doit être marié : cette obligation, inscrite dans la Discipline de l'Église de 1900, trouve son origine dans l'histoire de la mission et l'interdiction adressée aux premiers missionnaires britanniques d'épouser

1. Charles Forman note la forte implication des épouses de pasteurs et de missionnaires de la London Missionary Society dès la fin du XIX ${ }^{\mathrm{e}}$ siècle dans de nombreuses îles du Pacifique et notamment à Tahiti, les îles Cook, Samoa, Kiribati, Tuvalu et les îles Loyauté (1984 : 158-162).

2. École des hautes études en sciences sociales, juin 2005, sous la direction de Danièle Hervieu-Léger. La méthode retenue est celle d'entretiens semi directifs, réalisés entre octobre 2000 et août 2002 . 
des Tahitiennes, parce qu'elles n'étaient pas d'authentiques chrétiennes. Derrière l'interdiction de contracter des unions mixtes se cache une profonde défiance à l'égard des populations "indigènes " à convertir, particulièrement à l'égard des femmes tahitiennes perçues comme des tentatrices. Les récits des premières expéditions occidentales ont, en effet, contribué dès la fin du XVIII ${ }^{\mathrm{e}}$ siècle à forger le mythe de Tahiti et de la supposée liberté sexuelle qui y régnait ${ }^{3}$. Pour cette raison, dès 1806 le mariage avec une authentique chrétienne (une femme britannique) devient une condition préalable indispensable pour les missionnaires candidats à l'évangélisation à Tahiti (Davies, 1961: 91). Le mariage, érigé en instrument de moralisation et de stabilisation des relations conjugales, est par la suite exigé des Polynésiens souhaitant se convertir au protestantisme. En liant mariage chrétien et intégration dans la vie paroissiale des nouveaux mariés en tant que membres d'église ('etâretia), les missionnaires lient la fidélité conjugale à l'authenticité de la croyance des nouveaux convertis : il s'agit de prouver socialement, par un comportement conjugal jugé exemplaire, la profondeur de la foi chrétienne. À côté des deux sacrements reconnus dans le protestantisme, le baptême et la sainte cène, le protestantisme polynésien transforme donc le mariage - et avec lui la confirmation du baptême qui sont en principe célébrés dans un temps rapproché - en un quasi-sacrement. Au début des années 1960, l’Église décide d'assouplir les règles d'accès à la sainte cène : peuvent désormais la prendre des personnes confirmées mais pas mariées, c'est-à-dire qu'il devient possible d'accomplir la confirmation du baptême sans être marié. Cet assouplissement n'est toujours pas, quarante après, accepté dans toutes les paroisses.

Le pasteur est un homme marié qui sait combiner autorité et familiarité. Le mode de recrutement des pasteurs et la manière dont l'autorité pastorale est pensée sur le modèle de l'autorité parentale permettent de préciser les liens de familiarité qui unissent le pasteur et les fidèles. Les futurs pasteurs sont envoyés par leur paroisse d'origine à l'école pastorale. Jusque dans les années 1970, les pasteurs ainsi formés étaient renvoyés dans leur paroisse d'origine. Par la suite, un nouveau système de mutation pastorale oblige les pasteurs à changer de paroisse tous les trois ou six ans, alors que les diacres continuent à repérer parmi les jeunes paroissiens ceux qui feront de bons pasteurs et à espérer que les pasteurs ainsi formés retourneront un jour dans leur paroisse d'origine, ne serait-ce qu'à l'âge de la retraite. Les mutations pastorales introduisent une autre transformation dans l'exercice du ministère pastoral : jusque dans les années 1960, le pasteur était aussi agriculteur, le fait d'être muté dans sa paroisse d'origine lui permettant de cultiver les terres familiales. À partir des années 1970, le pasteur exerce sa charge à temps plein, dans une paroisse dont il n'est pas originaire, et n'étant plus agriculteur il dépend désormais entièrement de l'aide versée par l'Église pour subvenir à ses besoins et à ceux de sa famille.

3. Pour une lecture critique des récits des premiers explorateurs français (Bougainville et La Pérouse) : Tcherkézoff, 2005. 
Le pasteur ('Orometua) est considéré comme un metua (un parent, père ou mère) qui doit s'occuper de ses paroissiens et les aider à grandir spirituellement comme un parent s'occupe de ses enfants. Le terme utilisé pour l'adoption, $f a^{\prime} a^{\prime} a m u$, qui signifie littéralement "nourrir ", souligne qu'en Polynésie les liens de parenté s'expriment sur le registre nourricier. Comme le rappelle Alan Howard : "Oceanic terms for kinship imply, in one way or another, notions of common substance, or derivation from the same roots. The fundamental conception is that kinsmen share substance through common ancestry, but substance can also be shared by virtue of individuals being fed or nurtured from the same source " $(1996: 265)$. Les relations entre pasteur et paroissiens peuvent être analysées en termes d'échanges de nourritures qui participent à la création des liens de parenté. Le pasteur donne un enseignement ( $\left.h a^{\prime} a p i{ }^{\prime} i r a^{\prime} a\right)$ assimilé à une nourriture ( $\left.m \hat{a}{ }^{\prime} a\right)$ spirituelle. En échange, les paroissiens nourrissent leur pasteur en lui apportant taros, poissons et en l'invitant aux repas communautaires.

Le pasteur est aidé de son épouse qui, sans être consacrée ni rémunérée, participe activement à la vie de la paroisse en tant que simple membre d'église (laïque). Pourtant elle intervient à toutes les étapes de la "carrière " de son époux : les dossiers d'entrée à l'école pastorale comprennent l'évaluation de la culture générale (mathématiques, français) et/ou de la sincérité de la vocation pastorale des deux conjoints; depuis 1977, les femmes de pasteurs peuvent suivre les mêmes études de théologie que leurs époux; elles peuvent également, depuis les années 1990, soutenir un mémoire de fin d'année sanctionnant un travail de recherche personnel; elles bénéficient de bourses d'études octroyées par le Conseil œcuménique des églises (COE) ou la Communauté évangélique d'action apostolique (CEVAA) pour poursuivre, avec leurs maris, leur scolarité soit au Pacific Theological College à Fidji soit dans une faculté protestante de théologie en France, à Strasbourg ou à Montpellier; elles sont désormais associées à la cérémonie de consécration de leurs époux. Yvette Temauri, qui a suivi une formation théologique au Pacific Theological College avec son mari, fait partie de ces épouses de pasteurs qui ont revendiqué un autre rôle en paroisse :

«Avant, les femmes de pasteurs étaient très soumises, on a gratté un peu, et c'est aussi parce qu'en ce temps la formation n'était que pour les hommes et que les femmes n'avaient pas d'éducation, de formation pareille à l'homme et quand ils arrivent dans une paroisse, c'est l'homme qui prend la parole et la femme fait la popote, le mâ'a [nourriture] et s'occupe des enfants. Et moi je ne me dis pas comme les autres, quand je suis arrivée, je ne me suis pas laissée faire, c'est vrai une femme de pasteur est très respectée, mais à quoi ça sert d'être respectée si c'est seulement pour faire la popote ? Pour moi, aujourd'hui la femme du pasteur, c'est une femme qui aide son mari dans son travail et son ministère et qui aide les femmes de la paroisse. ${ }^{4}$

4. Yvette Temauri est entrée à l'école pastorale en 1966 en tant qu'épouse de pasteur. Au moment de l'entretien, en décembre 2001, son mari est retraité et elle est responsable du centre territorial d'information sur les droits de la femme et de la famille, organisme sous statut associatif dépendant des services du gouvernement de Polynésie française. 
Étudiant le rôle de la femme de pasteur, Bernard Reymond souligne que celleci peut jouer un rôle «sainement anticlérical » du fait de sa position de laïque et de sa non-participation aux instances décisionnelles de la paroisse ${ }^{5}$. En Polynésie française, si l'épouse est généralement considérée comme plus proche des paroissiens, plus accessible et accueillante, la question demeure de savoir si ces qualités sont liées à sa position de laïque, d'épouse ou à son sexe et pourquoi ces qualités devraient perdurer lorsque les femmes deviennent pasteures ou diacres. Enfin, certaines femmes de pasteurs ont pu transformer ce ministère dérivé en ministère à part entière en conservant leurs responsabilités au sein de l'Église alors même que leurs maris n'étaient plus pasteurs en activité (mari décédé, retraité ou démis pour cause d'adultère). Bien qu'exceptionnelle, la situation de ces femmes de pasteurs est parfaitement connue des paroissiens et surtout des paroissiennes du fait de leur action en faveur de l'institutionnalisation des activités des femmes et de l'accès des femmes au pastorat.

\section{L'institutionnalisation des activités paroissiales}

Au début des années 1980, la direction de l'Église encourage, dans chaque paroisse, la structuration des activités exclusivement réservées aux femmes, activités qui existaient, pour certaines d'entre-elles, dès les premiers temps de la mission mais n'avaient pas de caractère officiel et étaient vues davantage comme des lieux de socialisation que des lieux d'évangélisation. Cette reconnaissance dans l'organisation de la paroisse d'activités réservées aux femmes est directement liée à la participation de femmes de pasteurs, à partir des années 1970, aux différents organismes œcuméniques comme le Conseil œcuménique des églises au niveau mondial ou la Pacific Conference of Churches (PCC) au niveau régional. Ces organismes chargés de promouvoir le rapprochement entre les églises protestantes, catholiques et orthodoxes en travaillant à l'élaboration d'une liturgie commune sont efficacement engagés dans la lutte contre les discriminations sociales, économiques et les inégalités entre les sexes. Parce que le COE et la PCC ont demandé une représentation tripartite des Églises membres (un homme / une femme / un jeune) les femmes de pasteurs chargées de représenter les femmes de l'EEPF, et qui regrettaient lorsqu'elles participaient à ces réunions œcuméniques de ne représenter qu'elles-mêmes, ont demandé la mise en place au sein de chaque paroisse de Polynésie française de réunions régulières permettant d'une part de centraliser les demandes des femmes de l'Église et, d'autre part, de les informer en retour des activités œcuméniques et de la vie des autres Églises protestantes du Pacifique.

En Polynésie française, l'institutionnalisation des activités des femmes s'organise autour de trois axes: la mise en place d'un comité des femmes chargé de

5. Reymond citant Francine Dumas (1991 : 49-50). 
superviser l'ensemble de leurs activités (réflexions bibliques, ateliers couture, visites des malades et personnes âgées), l'organisation de rencontres des femmes des différentes paroisses ou églises, enfin la mise en place au sein de chaque paroisse d'un dimanche des femmes. Les deux premiers axes peuvent continuer à fonctionner avec l'organisation traditionnelle de la paroisse, en renforçant le rôle des épouses de diacres et l'efficacité du couple diaconal dans le premier cas et en organisant des rencontres de femmes à l'extérieur du temple, c'est-à-dire sans incidence directe sur l'organisation intra-paroissiale dans le second cas. Au contraire, la mise en place d'un dimanche de femmes crée les conditions d'une remise en cause radicale de l'organisation traditionnelle du culte dominical. Le dimanche des femmes signifie qu'une fois par mois, le plus souvent le troisième dimanche du mois après le dimanche de sainte cène et le dimanche des jeunes, le culte est organisé par les femmes de la paroisse, et la prédication peut être assurée par une femme. Alors que les activités des femmes étaient jusque-là organisées sur le mode d'une stricte séparation entre les sexes liant une prise de parole féminine à un auditoire féminin et un espace ordinaire, le dimanche des femmes opère un glissement doublement significatif en introduisant une parole féminine dans le temple et devant un auditoire mixte: les hommes doivent désormais écouter une femme parler. La question demeure de l'autorité que les paroissiens sont prêts à reconnaître à ce message : parole de Dieu ou simple parole de femmes, témoignage personnel qui n'engagerait qu'elles-mêmes ?

Dans un grand nombre de paroisses, la création d'un dimanche des femmes s'est accompagnée d'une restriction - en principe temporaire - qui en limite grandement la portée : seule l'épouse du pasteur (et éventuellement les épouses de diacres), c'est-à-dire celle qui a suivi les mêmes études de théologie que son mari, est autorisée à faire la prédication. Le dimanche des femmes devient dès lors l'occasion pour les paroissiens de juger qui, au sein du couple pastoral, est le prédicateur le plus brillant, la comparaison étant le plus souvent utilisée pour critiquer le pasteur ${ }^{6}$.

À un premier niveau, l'institutionnalisation des activités des femmes signifie la reconnaissance des femmes et de leur engagement dans la vie paroissiale, mais cette reconnaissance est limitée puisqu'elle se traduit par la constitution d'un comité de femmes restant entièrement dépendant du conseil des diacres, seule instance paroissiale habilitée à entériner les bilans et projets qui lui sont présentés. À un second niveau, ce processus marque une démocratisation de la prise de parole particulièrement saisissante quand les femmes réinvestissent le cadre paroissial selon un mode d'organisation qui leur est propre, en autorisant des femmes simples membres d'église à faire la prédication (et des jeunes filles qui

6. Le procédé n'est pas propre aux paroissiens de Polynésie française. P.-D. Dubeid souligne que si l'Église réformée de France a interdit aux couples de pasteurs d'exercer dans la même paroisse, c'est parce que « les dangers de concurrence dans la reconnaissance de la paroisse sont évidemment les plus forts lorsque l'activité des deux s'exerce pour le même public " (1990: 44). 
n'ont pas confirmé leur baptême à assurer la lecture biblique) alors que le culte dominical est traditionnellement, à travers la mise en scène des hiérarchies sociales, un temps de remise en ordre.

\section{D'un ministère dérivé à un " ministère à soi " "}

La décision du synode (ou conseil supérieur) de l'Église, en 1995, d'autoriser les femmes à devenir pasteures découle directement de la prise de position, quelques années auparavant, du grand comité des femmes de l'Église réunissant les femmes de pasteurs engagées dans les actions œcuméniques qui découvrent, dès le début des années 1980, que d'autres Églises du Pacifique ont consacré des femmes au ministère pastoral ${ }^{8}$. Emma Tufariua-Faua, épouse de pasteur qui a participé aux conférences de la Pacific Conference of Churches, se souvient de cette époque :

«On a travaillé sur le thème de la participation des femmes dans la société et dans l'Église. Et ça a été une période très enrichissante, pour voir comment les femmes abordaient leur rôle au niveau des Églises surtout. Et on découvrait qu'au niveau du Pacifique, certaines Églises, certaines îles, étaient bien plus avancées que nous, au niveau du ministère de la femme, par exemple aux îles Kiribati, il y avait déjà dix femmes consacrées, au Vanuatu, il y avait quatre femmes consacrées, en Papouasie, elles étaient quatre, à Fidji, elles étaient deux. Et en Nouvelle-Calédonie, il y avait déjà deux femmes consacrées en 1992, dont Marie-Claire [Kaemo] et nous, on était encore là... Le fait de trouver ça au niveau de la région et de dire que Tahiti, notre Église, n'était pas encore à ce stade-là, eh bien ça nous a motivées et on a lancé une réflexion au niveau du comité des femmes de l'Église, de toutes les paroisses (...). Au bout du compte, on était arrivé à faire, à dire que les femmes comprennent que les femmes, elles avaient autant de capacités pour ce ministère consacré. » ${ }^{9}$

En 1981, un premier cas avait été débattu, celui de Céline Hoiore, épouse de pasteur, qui avait révélé les tensions entre les différents échelons décisionnels de l'Église (paroisses/arrondissements/direction de l'Église) et des divergences quant à la définition même du rôle du synode tiraillé entre une stricte fonction de représentation des paroisses et une approche plus « avant-gardiste » défendue par les dirigeants de l'Église. Si Céline Hoiore n'a pas été consacrée pasteure en même temps que son mari, alors qu'elle était détentrice du même diplôme en théologie, c'est essentiellement parce que la question fut soulevée à une période où les épouses, désormais autorisées à suivre les mêmes études de théologie que leurs maris à l'école pastorale de Papeete, étaient toutes susceptibles de demander

7. L'expression est choisie en référence au livre de Virginia Woolf, Une chambre à soi.

8. Lors de la conférence de la Pacific Conference of Churches de 1981, une cérémonie est présidée par une femme pasteure de l'Église unie de Papouasie Nouvelle-Guinée et des îles Salomon.

9. Ancienne épouse de pasteur (séparée), Emma Tufariua-Faua est bibliothécaire et professeure à l'école pastorale de Hermon. Elle a 47 ans au moment de l'entretien en mai 2001. 
un jour à devenir, elles aussi, pasteures à part entière, mettant à mal le fonctionnement traditionnel du «couple pastoral».

En 1995, le synode autorise les femmes à devenir pasteures en précisant que rien dans la Bible n'interdit le pastorat des femmes. Cette décision n'a pas été l'objet de longs débats, le synode de 1995 étant occupé par ailleurs à dénoncer la reprise des essais nucléaires auxquels l'EEPF s'oppose officiellement depuis 1982 (Malogne-Fer, 2003). La position en faveur du pastorat des femmes ayant été qualifiée de "théologique ", les opposants ne pouvaient avancer que des arguments dits "culturels» et de facto disqualifiés. Les épouses de pasteurs sont beaucoup plus divisées que les hommes pasteurs, notamment parce que la situation des conjoint(e)s n'a pas été prise en compte. Alors que les femmes de pasteurs engagées dans le mouvement œcuménique sont satisfaites de cette décision, celles, plus nombreuses, qui exercent habituellement en paroisse se demandent comment une femme pourrait concilier vies privée et professionnelle si, dans le même temps, l'organisation paroissiale n'est pas modifiée. Les hommes pasteurs, au contraire, font preuve d'une unanimité quasiment sans faille : cette prise de position est pour eux une manière de renforcer leur statut de théologien puisque la question du pastorat des femmes est l'occasion de souligner qu'à la différence de la majorité des paroissiens, ils ont su assimiler une lecture «moderne » c'est-à-dire contextualisée de la Bible. Certains pasteurs demandent aux femmes d'être pasteures autrement et cette valorisation de la différence des sexes au service de l'institution ecclésiale est particulièrement forte chez ceux qui ont les plus hautes responsabilités à la tête de l'EEPF.

\section{Professionnalisation du pastorat}

Les mutations pastorales constituent une première étape de professionnalisation du ministère pastoral. Le pasteur n'est plus «pasteur-agriculteur »; envoyé dans une paroisse qui n'est plus sa paroisse d'origine, il n'est plus en mesure de cultiver les terres familiales. Devenu pasteur à plein temps, il doit désormais son autorité moins aux liens de parenté qu'aux enseignements reçus à l'école pastorale. Selon l'anthropologue Claude Robineau : "la tendance à ne plus nommer le pasteur dans sa paroisse d'origine correspondait à un souci de renforcer le statut théologique des pasteurs au détriment de leur insertion dans le monde profane. " (Robineau, 1994 : 237).

Cette transformation du ministère pastoral est à replacer dans un contexte de profondes mutations économiques et sociales. Depuis les années 1960 et l'installation du centre d'expérimentation du Pacifique à Moruroa et Fangataufa, l'économie polynésienne, auparavant essentiellement tournée vers les activités agricoles et la pêche, s'est rapidement tertiarisée. Le développement du secteur tertiaire, public et privé, fort pourvoyeur d'emplois «féminins " a favorisé la progression rapide de l'activité salariée des femmes ${ }^{10}$.

10. ISPF (Institut statistique de la Polynésie française), 2002. 
Avec l'arrivée des femmes au ministère pastoral se dessine une nouvelle légitimité que Jean-Paul Willaime (2002: 77) a mise en évidence en montrant comment, en France, la féminisation du corps pastoral participait à un processus de professionnalisation du ministère pastoral. La professionnalisation, écrit-il, s'analyse comme «le processus par lequel un métier s'autonomise par rapport à l'institution dans laquelle il s'exerce et s'auto-légitime à partir de la compétence qu'il met en œuvre. Le professionnel est un expert dans un domaine spécialisé et c'est sa qualité d'expert qui est la principale source de légitimité. Lorsque nous parlons de professionnalisation du pastorat, il s'agit aussi de rendre compte de l'évolution tendant à une nette séparation entre les tâches professionnelles et la vie privée du pasteur » (Willaime, $1986: 217$ ). La professionnalisation initiée par la direction de l'Église s'illustre à travers trois points : l'élévation du niveau d'études, l'émergence des ministères spécialisés et la dissociation progressive entre vie privée et vie professionnelle.

Dans le protestantisme français, l'accès des femmes au pastorat s'est effectué au début du $\mathrm{Xx}^{\mathrm{e}}$ siècle grâce aux facultés de théologie : "Avant qu'il y eut des femmes pasteurs, il y eut des étudiantes en théologie et des diplômées en théologie " (Willaime, 1996 : 36). En Polynésie française, les femmes entrent à l'école pastorale grâce à leurs diplômes : en 1995, l'Église autorise les femmes à devenir pasteures, en 1996, la direction de l'Église modifie les conditions d'entrée à l'école pastorale en instituant deux modes d'accès : un accès direct pour ceux qui ont le baccalauréat et un accès indirect appelé " année probatoire " pour les non bacheliers. Ces derniers doivent suivre pendant un an des cours du soir et passer à la fin de l'année scolaire un examen d'entrée à l'école pastorale d'autant plus sélectif que le nombre de candidats bacheliers aura été important. L'étude des parcours des hommes et des femmes entrés à l'école pastorale entre 1997 et 2001 montre que le niveau scolaire et la situation maritale se différencient sensiblement selon les sexes : les femmes sont quasiment toutes bachelières et célibataires, les hommes n'ont pas le baccalauréat et arrivent mariés à l'école pastorale.

La décision d'exiger le baccalauréat à l'entrée de l'école pastorale s'inscrit dans le cadre d'un développement important de la scolarisation en lycée et à l'université depuis une vingtaine d'années. L'université française du Pacifique a été créée en 1987, la scolarité jusqu'à 16 ans rendue obligatoire en 1992. Le pasteur étant, historiquement, un homme instruit - parfois aussi instituteur - la formation pastorale se devait de suivre cette tendance générale d'élévation du niveau scolaire. Mais l'exigence du baccalauréat est source de discussions et de mécontentements en paroisse car cette mesure est perçue comme trop favorable aux jeunes filles, qui réussissent mieux leur scolarité que les garçons. La question des ressorts de la vocation pastorale, entre connaissances scolaires et foi personnelle, sert dès lors à discréditer la démarche de ces jeunes filles présentée comme un risque d'intellectualisation du ministère pastoral.

Si la qualification théologique est primordiale dans l'exercice du ministère pastoral, l'arrivée des femmes à l'école pastorale fait donc ressurgir une opposition 
interne au protestantisme entre l'accession à la vérité divine par les connaissances ou l'expérience. L'historien Jean-François Zorn a montré comment, au XIX ${ }^{\mathrm{e}}$ siècle, la question de la formation des pasteurs et des missionnaires a réactivé des tensions entre les différents courants théologiques du protestantisme: les facultés de théologie protestante visaient à former les pasteurs tandis que l'école des missions était chargée, sous l'impulsion de la société des missions évangéliques de Paris proche de la "théologie du Réveil », de former les futurs missionnaires destinés à l'Outre-mer et notamment à Tahiti (Zorn, 2004 : 13-21). Si l'EEPF est membre de l'Alliance réformée mondiale, l'histoire des missions britannique puis française et la présence de missionnaires de tendances théologiques différentes créent les conditions d'une valorisation de l'expérience allant de pair avec une critique des études scolaires et universitaires.

La critique exprimée sous forme interrogative, "la vocation est-elle une question de foi ou de diplômes ? ", construit une opposition étanche entre l'authenticité de la foi chrétienne et les connaissances scolaires qui " assècheraient » la foi. Cette critique formulée par des paroissiens mécontents s'adresse directement aux jeunes femmes, suspectées de ne pas avoir grandi en paroisse d'une part parce que la forte concentration des lycées à Papeete oblige les élèves qui veulent poursuivre leurs études à quitter l'île ou la paroisse d'origine et d'autre part parce que l'exigence du baccalauréat signifie a contrario la fin du monopole détenu jusque-là par les diacres en matière de discernement des vocations pastorales. Et parce que les jeunes filles ne peuvent pas être envoyées à l'école pastorale par des diacres qui continuent à privilégier des candidatures d'hommes mariés, elles ne peuvent pas avoir "grandi » en paroisse. Derrière la réactivation d'une opposition entre les diplômes et la foi se dessinent des tensions entre deux modes d'enseignement, l'un dispensé à l'école de la République française et validé par des diplômes, l'autre dispensé en paroisse dans le cadre des activités de l'école du dimanche, des réflexions bibliques et des prédications.

La logique scolaire crée tout à la fois une démocratisation des conditions d'entrée à l'école pastorale en acceptant des candidats - les femmes - qui en étaient jusque-là exclus, et une nouvelle hiérarchisation parmi les pasteurs en exercice, selon le niveau des diplômes obtenus, la réputation de la faculté et l'implication dans la commission d'animation théologique. Les pasteurs en paroisse membres de la commission d'animation théologique illustrent particulièrement bien l'émergence de la figure du pasteur-théologien, qui ajoute à l'expérience paroissiale un nouveau type de légitimité, acquis grâce aux enseignements dispensés par un docteur en linguistique, Turo Raapoto, fils de Samuel Raapoto, qui fut le premier pasteur polynésien président de l’Église évangélique devenue autonome en $1963^{11}$. Cette dynamique profondément élitiste permet une recléricalisation en même temps que se poursuit la professionnalisation du ministère pastoral. L'engagement dans

11. L'autonomie de l'Église en 1963 signifie que cette dernière est désormais dirigée par des pasteurs polynésiens et non par des missionnaires français de la société des missions évangéliques de Paris. 
la commission d'animation théologique, qui contribue à la recléricalisation du ministère pastoral, n'est pas chez les hommes pasteurs une réaction à la féminisation du corps pastoral, qu'ils souhaitaient pour la plupart, c'est le même processus, celui de la professionnalisation du ministère pastoral, qui produit des effets différenciés selon les sexes parce qu'il s'inscrit dans un cadre traditionnel de socialisation et de division sexuées du travail. L'articulation entre le savoir et le pouvoir ne s'opère pas de la même façon selon les sexes : les élèves pasteures se destinent pour la plupart à exercer un ministère spécialisé, en marge du pouvoir institutionnel. En reprenant la distinction opérée par Nicole Mosconi (1994) à propos de l'éducation des femmes, entre accession aux savoirs, transmission des savoirs et création de nouveaux savoirs, il apparaît que les femmes ont eu accès au savoir biblique dès l'implantation des missions anglaises et françaises, elles ont également accès aux fonctions de transmission du savoir (elles représentent aujourd'hui les deux tiers des moniteurs de l'école du dimanche), en revanche, elles n'ont pas accès à la création de nouveaux savoirs.

Les ministères spécialisés visent une population qui n'est pas définie en fonction d'une appartenance territoriale et qui ne fréquente pas de paroisse: les jeunes dans les écoles, les malades dans les hôpitaux, les prisonniers, etc. L'émergence de ces ministères participe à une remise en cause du cadre paroissial comme modèle exclusif d'organisation ecclésiale dans un contexte de fortes migrations - des archipels vers Tahiti - et d'urbanisation croissante à Tahiti qui rassemble désormais $70 \%$ de la population (ISPF, 2003). Les ministères spécialisés sont, pour beaucoup, des ministères qui conviendraient particulièrement bien aux femmes pasteures pour au moins deux raisons. La première est purement négative : l'acceptation des femmes pasteures en paroisse ne va pas de soi et risque de poser beaucoup de difficultés, les ministères spécialisés permettent de résoudre l'impossible équation entre la décision synodale autorisant les femmes à accéder au pastorat et les fortes réticences des paroissiens; la seconde raison est que les ministères spécialisés - comme pasteur aumônier des écoles ou des hôpitaux correspondent à des tâches et des secteurs d'activités (éducation des jeunes enfants et soins apportés aux malades) traditionnellement dévolus aux femmes. Ainsi, parmi les quatre premières femmes pasteures stagiaires, une seule a été affectée dans une paroisse traditionnelle : les trois autres ont effectué leurs stages dans les services administratifs de l'Église, ou dans l'une des deux paroisses extraterritoriales que compte l'EEPF : les paroisses de langues française et chinoise de Papeete. La première femme pasteure de l'Église est aujourd'hui aumônière au lycée protestant de Arve, renouant ainsi avec son ancien métier d'institutrice.

Enfin, les ministères spécialisés permettent plus facilement que les ministères paroissiaux une séparation entre vies «professionnelle » et «privée » à laquelle beaucoup de femmes aspirent. Le pasteur habite avec sa famille dans un presbytère qui est le plus souvent attenant au temple, les paroissiens viennent fréquemment soit aider au ménage lorsqu'il s'agit d'accueillir des invités de la paroisse qui logent au presbytère, soit emprunter des ustensiles de cuisine pour préparer des repas paroissiaux. Depuis quelques années, et notamment sous l'impulsion de 
femmes de pasteurs qui souhaitent plus d'intimité pour elles et leurs enfants, la revendication d'une séparation entre vie privée et vie professionnelle se fait jour. C'est notamment le cas de Line Tehahe :

"Les femmes [de] pasteurs d'avant, comme [ma belle-mère], elles sont gentilles comme tout, elles accueillent les gens. On fait toujours grand le presbytère, c'est pour accueillir les gens de passage. Celui qui est là avant, les gens avaient l'habitude de rentrer dans le presbytère pour chercher un couteau, un balai, de la vaisselle. Et après c'est à toi à aller courir derrière pour récupérer! Moi, j’ai dit non. Je pense qu'il y a un respect aussi. » ${ }^{12}$

Les pasteurs n'hésitent plus à prendre quelques jours de vacances, les femmes de pasteurs sont de plus en plus nombreuses à vouloir travailler à l'extérieur de la paroisse. Avec l'arrivée des femmes au ministère pastoral, la dissociation devrait être menée à terme pour protéger le mari des sarcasmes des paroissiens.

Le schéma ci-dessous reprend les différents types de ministères pastoraux en les situant dans le cadre de deux dynamiques en partie contradictoires: une professionnalisation du ministère qui remet en cause les figures $\mathrm{du}$ "couple pastoral » et du pasteur-agriculteur et une démocratisation de l'accès à la parole qui autorise les femmes à assurer la prédication et à devenir pasteures mais qui n'a pas atteint les lieux de production théologique.

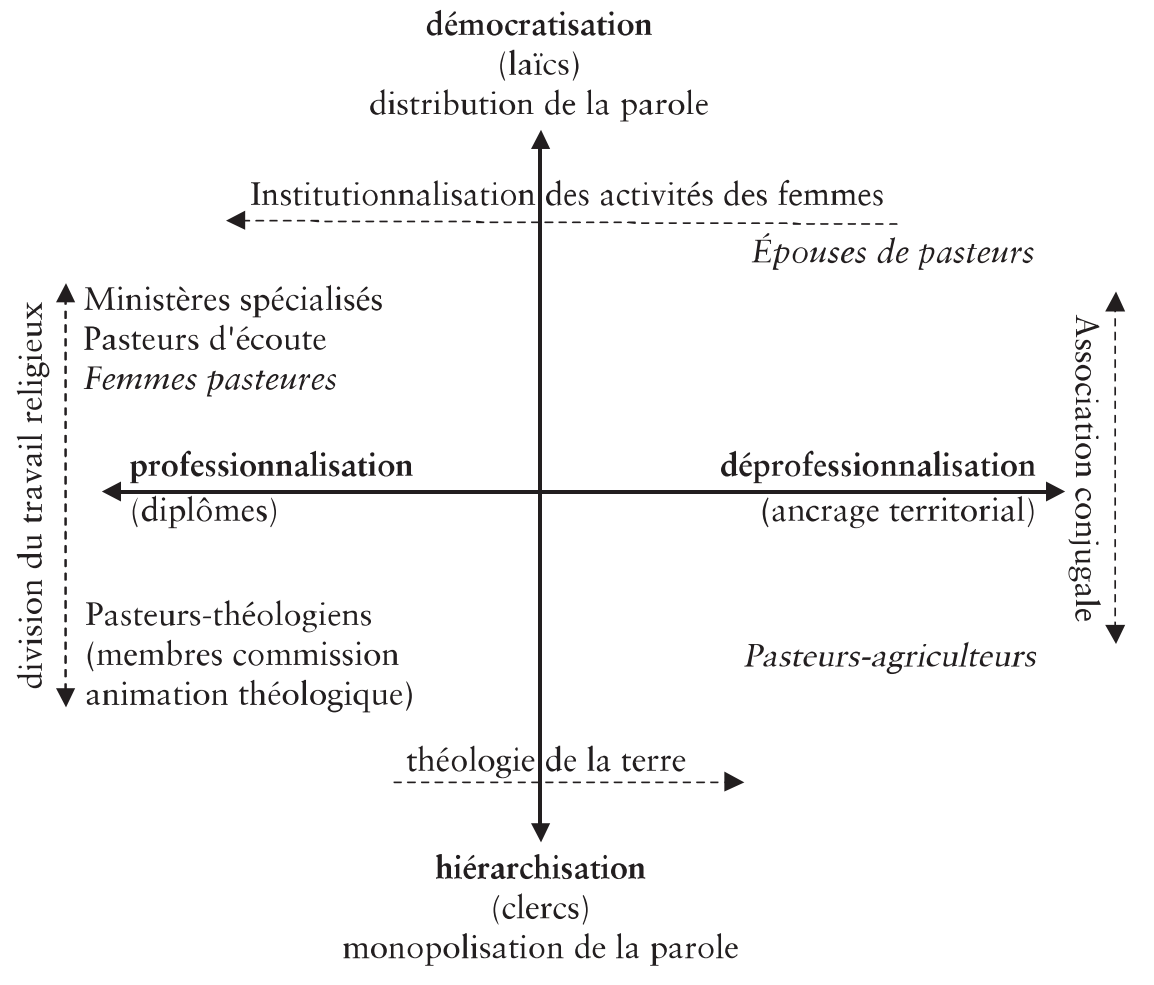

12. Entretien de novembre 2001. 


\section{Reconfigurations conjugales}

En 1995, l'EEPF autorise les femmes à devenir pasteures tout en maintenant implicitement l'obligation de mariage et en refusant que les deux conjoints au sein d'un même couple puissent être tous les deux consacrés pasteurs, créant de fait une situation originale où la place en paroisse et en famille de celui qui n'est que «mari de pasteure " est l'objet de toutes les interrogations. Lorsque les femmes arrivent à cette fonction, les paroissiens n'interrogent pas la pertinence de l'organisation paroissiale mais celle de la décision synodale autorisant les femmes à accéder au pastorat : parce que la paroisse ne prévoit rien pour le mari de la femme pasteure, cette difficulté à définir la place du mari se transforme en incapacité à imaginer une femme pasteure. D'une certaine façon (mais d'une certaine façon seulement) l'institutionnalisation des activités des femmes prépare la paroisse au pastorat des femmes, même si, dans le même temps, le fait que les femmes aient désormais "leur place » au sein de la paroisse est un argument de poids fréquemment repris par les opposants au pastorat des femmes qui regrettent que celles-ci en "veuillent toujours plus ", un comportement qu'ils jugent peu «évangélique».

Lorsque l'homme est pasteur, son épouse a des activités et des responsabilités au sein de la paroisse non officiellement reconnues mais qui sont néanmoins considérées comme indispensables à la bonne marche de la paroisse : présidence du comité des femmes, animation des réflexions bibliques destinées à adapter le message biblique à la situation concrète des femmes, organisation de tournées mensuelles de maisons à destination des personnes âgées ou malades.

La femme du pasteur ne participe pas à l'instance décisionnelle de la paroisse qui est le conseil de diacres, composé exclusivement des diacres et présidé par le pasteur. Elle informe néanmoins son mari, en tant que présidente du comité des femmes, des projets du comité qui doivent obtenir l'aval du conseil de diacres et, de façon plus générale, de ce qui se passe "du côté des femmes ». La femme a donc un pouvoir de représentation, mais uniquement de façon officieuse auprès de son mari, et ne participe pas au pouvoir décisionnel. En revanche, le pasteur peut, grâce aux informations collectées auprès de son épouse, devenir en conseil de diacres le porte-parole de toute la paroisse, des hommes comme des femmes.

La même dynamique s'observe au niveau de la fonction de diacre. La paroisse est divisée en 'âmuira'a, ou groupes, rassemblant les paroissiens par origine familiale et territoriale : les 'âmuira'a sont dirigés par des diacres, qui, à leur niveau, sont aussi des hommes d'autorité et des hommes mariés et ont capacité à représenter les paroissiens du 'âmuira'a, les hommes comme les femmes, et à parler en leur nom grâce à l'implication des femmes de diacres dans le comité des femmes du 'âmuira'a et de la paroisse.

La façon dont s'organise la paroisse, avec des moments comme le culte où hommes et femmes se côtoient et des périodes où les activités sont séparées selon 
les sexes, justifie la présence à sa tête de couples pastoraux et diaconaux : la structuration de la paroisse en deux côtés, qui ne peut fonctionner qu'avec un couple pastoral, est un cas typique de ce que Erving Goffman désigne sous le terme de réflexivité institutionnelle. Partant de la question suivante " comment, dans une société moderne, ces différences biologiques non pertinentes entre les sexes en viennent-elles à sembler d'une telle importante sociale ? Comment sans justification biologique, ces différences biologiques sont-elles élaborées socialement ? " (2002: 89), Goffman définit la réflexivité institutionnelle comme un mode de représentation des différences des sexes qui consiste à faire de l'organisation institutionnelle "une conséquence naturelle de la différence entre les classes sexuelles, alors qu'en fait c'est plutôt un moyen d'honorer, sinon de produire, cette différence» (2002: 79-82).

Lorsque la femme devient pasteure, tout devient problématique, y compris sa vie conjugale et familiale. L'organisation paroissiale fonctionne comme une structure de mémorisation du modèle antérieur (un homme pasteur aidé de son épouse) produisant des effets de dissonance institutionnelle (Singly et Chaland, $2002: 151)^{13}$. Les jeunes femmes entrent à l'école pastorale célibataires et grâce au baccalauréat, mais parce que la paroisse continue à réclamer des pasteurs mariés, ces élèves pasteures sont invitées à se marier rapidement, sinon l'Église se chargera de leur trouver un mari. Elles considèrent qu'elles n'ont pas besoin d'un mari pour étudier, bien au contraire. La vie conjugale et familiale de ces futures femmes pasteures est l'objet de toutes les discussions: lorsqu'elles sont présentées en paroisse, c'est d'abord comme célibataires, à la recherche d'un mari, et ensuite comme élèves pasteures.

Mais lorsqu'elles sont mariées, la situation n'est pas plus enviable: une femme accédant au pastorat "pique " la place de son mari et, ce faisant, lui manque de respect, et parce qu'elle manque de respect à son mari, elle manquera de respect aux hommes de la paroisse, à leurs épouses et de proche en proche à l'ensemble de la paroisse. La question «comment une femme qui ne respecte pas son mari pourrait-elle respecter la paroisse? » montre de quelle façon la cellule familiale et conjugale sert à disqualifier l'accès des femmes au pastorat. Lorsque l'homme est pasteur, l'autorité au sein de la famille sert de modèle aux paroissiens, alors que la femme dans la même fonction ne devrait pas se servir de son autorité au sein de la paroisse et du couple pour "rabaisser " son mari.

Chez les élèves pasteures un compromis semble se dessiner progressivement : un mariage retardé au maximum et des exigences minimales concernant le futur mari à qui il est simplement demandé d'accepter que son épouse devienne pasteure. Comme elles retardent leur mariage, le futur mari n'aura pas de formation

13. Le terme de dissonance institutionnelle est emprunté à François de Singly et Karine Chaland qui, à partir d'une étude des femmes préfètes, mettent en évidence la gestion de l'inversion des positions habituelles qui passe par une moindre participation du conjoint aux cérémonies et autres réceptions sources de dissonance institutionnelle. 
théologique lui permettant de participer légitimement aux activités paroissiales et d'aider son épouse dans l'exercice du ministère pastoral. La situation du mari de la pasteure concentre donc toutes les interrogations et beaucoup de paroissiens refusent l'accession des femmes au ministère pastoral au motif qu'ils ne sauraient pas comment accueillir son mari, ni quelles seraient ses responsabilités dans la paroisse. La question la plus fréquente est : qui va s'occuper des femmes de la paroisse, la femme pasteure en tant que femme ou son mari en tant que conjoint ?

L'accession des femmes au pastorat est l'occasion de mettre en lumière des compréhensions différentes selon les sexes: alors que les hommes pasteurs interrogés n'imaginent pas que le pasteur, homme ou femme, puisse un jour être célibataire et rappellent que le conjoint (c'est-à-dire leur conjointe) est une aide, "la meilleure des confidentes ", les femmes de pasteurs sont quelques-unes à avoir une vision beaucoup moins enchantée des relations maritales et n'hésitent pas à souhaiter qu'un jour la femme pasteure puisse rester célibataire pour être libre de ses faits et gestes. De même que les hommes pasteurs n'envisagent pas qu'un époux de pasteure puisse un jour présider le comité des femmes, les épouses de pasteurs sont beaucoup plus nombreuses à penser qu'un mari qui a suivi des études de théologie pourrait participer en tant qu' " enseignant » aux activités des femmes. Dans le premier cas, le point de vue des hommes pasteurs, les réflexions bibliques des femmes sont vues comme un simple lieu de sociabilité, dans le second cas, le point de vue des femmes de pasteurs, les réflexions bibliques sont d'abord un lieu d'enseignement.

Le même mécanisme de dissonance institutionnelle est également observable chez les femmes diacres. Si l'Église accepte que des femmes puissent être installées diacres depuis une cinquantaine d'années, ce n'est qu'au cours des années 1990 que la situation des femmes diacres a cessé d'être exceptionnelle. Parmi les trentetrois femmes diacres rencontrées, la plupart d'entre elles sont devenues diacres assez tardivement, en moyenne à l'âge de 40 ans (contre 37 ans pour les hommes) ${ }^{14}$. Elles n'ont, le plus souvent, jamais quitté la paroisse, en effectuant la confirmation du baptême dès l'adolescence (sans attendre de se marier) et en devenant très vite monitrices ou aide-monitrices de l'école du dimanche dès qu'elles n'avaient plus l'âge de rester à l'école du dimanche en tant qu'élèves. La confirmation du baptême, contre l'avis des parents - attachés à une compréhension traditionnelle de la confirmation comme preuve de sagesse et de maturité sociale - est présentée comme le premier acte d'émancipation de la tutelle parentale. À l'inverse, pendant leur adolescence, les hommes ont tendance à s'absenter pour une période plus ou moins longue des activités paroissiales et ne recommencent à s'y impliquer que lorsqu'ils deviennent officiellement diacres. Dans le premier cas, le titre de diacre intervient comme une reconnaissance du travail déjà accompli, en

14. L'estimation chez les hommes diacres est fondée sur les résultats d'un questionnaire distribué entre janvier et août 2002 dans les paroisses de Tahiti et des îles Australes (trente réponses valides). 
d'autres termes c'est parce que les femmes ont déjà fait leur preuve qu'elles sont acceptées au sein de la paroisse. Dans le second cas, c'est le titre qui autorise et oblige le diacre nouvellement installé à s'impliquer dans les activités paroissiales : à la différence des femmes, les hommes « héritent " le plus souvent d'une charge diaconale qui, dans le même temps, leur laisse peu de marge de manœuvre, le diacre retraité restant à leur côté pour leur apprendre le travail de diacre et les surveiller.

Parmi les trente-trois femmes diacres rencontrées, dans trois cas sur quatre, les femmes deviennent diacres parce que leur mari n'a pas pu accéder au diaconat, généralement parce qu'il n'est pas lui-même polynésien et/ou protestant pratiquant. La situation la plus fréquente est celle d'une femme polynésienne mariée à un homme demi (c'est-à-dire métis) ou d'origine chinoise : les paroissiens préfèrent être dirigés par une femme polynésienne que par quelqu'un considéré comme étranger, les relations paroissiales étant pensées sur le modèle des relations familiales et parentales. C'est cette pratique moindre ou inexistante du conjoint qui explique l'accession des femmes au ministère diaconal mais c'est précisément cette absence de pratique religieuse qui, par la suite, peut entraver la femme diacre dans l'exercice de son ministère. Alors que la paroisse exige traditionnellement des deux conjoints les mêmes exemplarité comportementale et rigueur morale, la femme diacre fait l'expérience, parfois douloureuse, de devoir répondre de ses actes, mais aussi de son mari, si celui-ci n'a pas un comportement religieux irréprochable. Léontine, diacre de la paroisse de Tevaitoa (Raiatea) reconnaît qu'au début, son mari demi ne venait jamais au culte :

«Il aimait bien aller avec les jeunes, voir les combats de coqs. Quand je faisais des sermons, mon mari, je le voyais passer devant pour aller au combat de coqs, alors j'étais critiquée, elle nous fait le sermon et son mari ne va pas au purera'a! [culte]. " ${ }^{15}$

Mais le célibat des diacres reste largement impensable et dans les rares cas où des femmes célibataires deviennent diacres, il leur est reproché de vouloir commander les paroissiens alors qu'elles n'ont pas l'expérience des relations conjugales et parentales. Virginie a 44 ans, elle est diacre de la paroisse de Mahina (Tahiti) et célibataire :

"Quand on m'avait dit, il faut que tu te maries avant d'être diacre, c'est les femmes qui m'ont dit, j’ai regardé vers Jésus et j’ai dit, “et lui alors ? il n'est pas marié !” "Oui, mais lui c'est pas pareil..." Je crois c'est la première fois dans l'Évangile, une femme célibataire. Dans le 'âmuira'a, ils me disent, "tu n'as pas de mari, tu n'as pas d'enfants et tu viens nous commander ?" Je réponds, "c'est vrai, mais je suis responsable de vous et quand on est diacre, on est parent de ce 'âmuira'a", ils disent "c'est pas normal”, oh laisse tomber... " ${ }^{16}$

La vie familiale et conjugale de la femme diacre ou pasteure n'est plus le reflet d'une vie exemplaire qui sert à la fois de modèle pour les paroissiens et de

15. Léontine, institutrice retraitée, a 61 ans au moment de l'entretien en juin 2001.

16. Entretien de juillet 2001. 
condition d'accès à des responsabilités au sein de la paroisse. La légitimité par les diplômes, dans le cas des femmes pasteures et par le travail, dans le cas des femmes diacres, apparait a posteriori comme une façon de compenser ce défaut de crédibilité dû à une situation conjugale inhabituelle.

La féminisation du corps pastoral et diaconal intervient dans un contexte colonial en forte mutation, les revendications d'égal accès des femmes à des fonctions de représentation et de décision détenues jusque-là par des hommes sont vite reléguées à des questions secondaires voire profondément contraires à l'identité et l'authenticité polynésiennes: la libération du peuple mâ'ohi pour laquelle l'Église protestante est fortement engagée ne saurait admettre d'autres formes de revendications en interne. La féminisation du ministère pastoral participe à l'émergence d'un nouveau type de légitimité, acquise par les diplômes, contribuant à faire du pasteur un théologien plutôt qu'un agriculteur. Mais dans le même temps tout se passe comme si les femmes participaient à l'émergence d'un nouveau type de légitimité auquel elles n'auront jamais pleinement accès, dynamique illustrée notamment par les modalités de fonctionnement et la production théologique de la commission d'animation théologique qui regroupe des pasteurs exerçant en paroisse et prône le retour à la terre et à la langue mâ'ohi comme seule voie de salut.

L'institutionnalisation des activités des femmes au sein des paroisses s'est traduite par une démocratisation de l'accès à la parole: plus besoin d'être consacré pasteur ou installé diacre pour prendre la parole dans le temple. Une des conséquences de cette institutionnalisation a été la revendication, sous l'impulsion des épouses de pasteurs impliquées dans le mouvement œcuménique, de l'accession des femmes au pastorat. Mais lorsque les femmes deviennent pasteures, le modèle de référence qui continue à vouloir fonctionner est celui d'un homme marié, en d'autres termes la démocratisation de l'accès au savoir et à la prédication ne s'est pas accompagnée d'une redéfinition des critères servant à définir ce qu'est un «bon pasteur ».

Gwendoline MALOGNE-FER

Groupe Sociétés, religions, laïcités

\section{Bibliographie}

Davies John, The History of the Tahitian Mission, 1799-1830, C. W. Newbury, ed., Cambridge University Press, 1961.

DubeID Pierre-Luigi, Le pasteur : un interprète, Genève, Labor et Fides, 1990.

ERNST Manfred, «Les groupes religieux à croissance rapide en Polynésie française : implications pour les principales Églises et la société ", Les Cahiers du Veà Porotetani, suppl. au n 17 (Actes du colloque international des 7 et 8 mars 1997), Papeete, 1997, pp. 83-88. 
Fer Yannick, Pentecôtisme en Polynésie française, l'Évangile relationnel, Genève, Labor et Fides, 2005.

Fer Yannick et Malogne-Fer Gwendoline, "Christianisme, identités culturelles et communautés en Polynésie française » in La France et les outre-mers, l'enjeu multiculturel, Paris, CNRS Éditions, Hermès, pp. 355-365.

Forman Charles, "Sing to the Lord a New Song: Women in the Churches of Oceania ", in O'Brien Denise et SHaron W. Tiffany (dirs.), Rethinking Women's Roles. Perspectives from the Pacific, Berkeley, University of California Press, 1984, pp. 153-172.

Goffman Erving, L'arrangement des sexes, Paris, La Dispute, 2002.

Howard Alan, "Cultural Paradigms, History, and the Search for Identity in Oceania ", in LINNEKIN Jocelyne et POYER Lin (eds.), Cultural Identity and Ethnicity in the Pacific, Honolulu, University of Hawai'i Press, 1990, pp. 259-279.

ISPF (Institut statistique de la Polynésie française) et ministère polynésien du tourisme, de l'environnement et de la condition féminine, Vahine en chiffres, Papeete, 2002.

ISPF, Points forts $n^{\circ}$ 4/2003, Papeete, 2003.

MALOGNE-Fer Gwendoline, "L'EEPF et les essais nucléaires : de la prise de conscience chrétienne à la prise de position publique (1963-1982)" in Jean-Marc Regnault (dir.), François Mitterrand et les territoires français du Pacifique (1981-1988), Paris, Les Indes Savantes, 2003, pp. 205-214.

-, Quand les femmes prennent la parole : démocratisation institutionnelle et professionnalisation des ministères au sein de l'église évangélique de Polynésie française, thèse de doctorat en sociologie, Paris, EHESS, 2005.

-, Les femmes dans l'Église protestante mâ'ohi: Religion, genre et pouvoir en Polynésie française, Paris, Karthala, à paraître 2007.

Mosconi Nicole, Femmes et rapport au savoir. La société, l'école et la division sexuelle des savoirs, Paris, L'Harmattan, 1994.

SAURA Bruno, "Théologie de la libération et théorie de la culture chez Duro Raapoto ", Bulletin de la société des études océaniennes, 246, Papeete, 1989, pp. 1-15.

Singly François de et Chaland Karine, "Avoir "le second rôle" dans une équipe conjugale », Revue française de sociologie, 43-1, 2002, pp. 127-158.

Reymond Bernard, La femme du pasteur. Un sacerdoce obligé? Genève, Labor et Fides, 1991.

Robineau Claude, Tradition et modernité aux îles Sous-le-Vent, livre I, Du coprah à l'atome, Paris, Éditions de l'ORSTOM, coll. « Mémoires », 100, 1984.

TCherkezoff Serge, "La Polynésie des vahinés et la nature des femmes : une utopie occidentale masculine ", CLIO, Histoire, femmes et sociétés, 22, 2005, pp. 63-82.

Willaime Jean-Paul, Profession: pasteur. Sociologie de la condition du clerc à la fin $d u$ XX ${ }^{e}$ siècle, Genève, Labor et Fides, 1986.

WiLlaime Jean-Paul, "Les pasteures et les mutations contemporaines du rôle de clerc ", CLIO, Histoire, femmes et sociétés, 15, 2002, pp. 69-83.

WiLlaime Jean-Paul, "L'accès des femmes au pastorat et la sécularisation du rôle du clerc dans le protestantisme ", Archives de sciences sociales des religions, 95, Paris, 1996, pp. 29-45.

ZoRN Jean-François, La missiologie, émergence d'une discipline théologique, Genève, Labor et Fides, 2004. 


\section{Annexe}

Repères chronologiques sur l’Église évangélique de la Polynésie française

1797 : Arrivée des premiers missionnaires protestants de la London Missionary Society le 5 mars 1797 à Tahiti.

1863 : Arrivée du premier missionnaire français à Tahiti, Thomas Arbousset.

1963 : L'Église accède à l'autonomie c'est-à-dire qu'elle n'est plus dirigée par des missionnaires français mais par des pasteurs polynésiens.

1966 : Création du Pacific Theological College à Fidji destiné à accueillir les futurs pasteurs originaires des églises du Pacifique.

1977 : Les épouses de pasteurs sont autorisées à suivre les mêmes cours que leurs maris à l'école pastorale de Hermon (Papeete).

1989 : Publication du premier livre de la commission d'animation théologique édité par l'Église évangélique de Polynésie française intitulé : Te rautira'a i te parau a te Atua e te iho tumu mâ'ohi ou L'animation de la parole de Dieu et la nature originelle mâ'ohi.

1995 : Décision synodale autorisant les femmes à devenir pasteures.

2004 : L’Église évangélique de Polynésie française est rebaptisée Église protestante mâ’ohi au cours du synode d'août 2004.

\section{Résumé}

Le fil conducteur de cette étude est d'interroger, à partir du terrain protestant polynésien, la transformation de l'institution ecclésiale en lien avec la transformation des relations entre les hommes et les femmes en paroisse. L'institutionnalisation des activités des femmes initiée par les épouses de pasteurs depuis les années 1980 et l'accession des femmes au ministère pastoral en 1995 correspondent à deux dynamiques - parfois concurrentes - de participation accrue des femmes au sein de l'Église évangélique de Polynésie française. Avant que les femmes aient pu devenir diacres ou pasteurs, l'Église a été un lieu de sociabilité apprécié par les femmes qui ont su organiser des lieux et des moments exclusivement féminins.

L'accès des femmes au pastorat illustre une professionnalisation des ministères pastoraux qui signifie l'accent mis sur la formation théologique des élèves pasteurs, la création de ministères spécialisés non paroissiaux et enfin la séparation entre vie privée et vie professionnelle. Mais alors que les relations en paroisse sont pensées sur le mode des relations parentales (le pasteur est un parent qui doit aider les paroissiens à grandir spirituellement), la mobilisation de la sphère privée, c'est-à-dire la constitution de la famille du pasteur en modèle, s'opère différemment selon le sexe du pasteur.

Mots-clés : femmes, pastorat, protestantisme, Polynésie française. 


\section{Abstract}

The main theme of this study consists in questioning, from the Polynesian Protestant field, the connection between the transformation of the church institution and the transformation of men-women relations in parish. The institutionalization of women's activities initiated during the 1980's by pastor's wives and the acceptance of woman pastorate in 1995 show two distinct - and sometimes conflicting - processes increasing the participation of women in the church life. Before women became deacons or pastors, the church has been a place of socialization appreciated by women who have organized places and times for women only.

The accession of women to pastoral ministry ges with a professionalization of ministries characterized by a priority given to the theological training of the future pastors, the creation of specialized non-parish ministries and the separation between private life and professional life. But while the relations within the parish are considered in terms of parental relations (the pastor is a parent who should facilitate the spiritual growth of his parishioners), the mobilization of the private life, i.e. the use of the pastor's family as an example, does not work for a woman pastor as it does for a man pastor.

Keys words: women, pastorate, protestantism, Polynesia.

\section{Resumen}

El hilo conductor de este estudio es, a partir del terreno protestante polinesio, la tranformación de la institutión eclesial, vinculada con la transformación de las relaciones entre hombres y mujeres en la parroquia. La institucionalización de las actividades de las mujeres, iniciada por las esposas de los pastores desde los años 80 y el acceso de las mujeres al ministerio pastoral en 1995, corresponden a 2 dinàmicas - a veces contrarias - de mayor participación de las mujeres en el seno de la Iglesia Evangélica de Polinesia Francesa. La Iglesia, antes de que las mujeres hayan podido llegar a ser diàconos o pastores, fue un lugar de sociabilidad apreciado por las mujeres.

El hecho de que las mujeres llegan a ser pastores ilustra la profesionalización de los ministerios pastorales es decir que han hecho hincapié en la formación teológica de los alumnos pastores, la creaciòn de ministerios especializados non-parroquiales y por fin, la separaciòn entre vida privada y vida profesional. Pero mientras que las relaciones en parroquia estàn pensadas sobre el modo de las relaciones de parientes (el pastor es como un padre que ayuda a sus feligreces a elevarse espiritualmente) la movilizaciòn de la esfera privada, es decir la constituciòn de la familia del pastor en un modelo, se opera diferentemente segùn el sexo del pastor.

Palabra clave: mujeres, ministerio de pastores, protestantismo, Polinesia Francesa. 